\title{
The Vpu-interacting protein ATP6V0C regulates expression of tetherin and HIV-1 release
}

\author{
Abdul A. Waheed ${ }^{* 1}$, Maya Swiderski ${ }^{1, \#}$, Ali Khan ${ }^{1, \#}$, Ariana Gitzen ${ }^{1}$, Ahlam Majadly ${ }^{1}$, and \\ Eric O. Freed ${ }^{1}$
}

${ }^{1}$ Virus-Cell Interaction Section, HIV Dynamics and Replication Program, Center for Cancer

Research, National Cancer Institute, Frederick, MD 21702

Running title: ATP6V0C is required for Vpu-mediated tetherin degradation

${ }^{\#}$ These authors contributed equally to this work

${ }^{*}$ To whom correspondence should be addressed: Abdul Waheed, Virus-Cell Interaction Section, HIV Dynamics and Replication Program, National Cancer Institute, Frederick, MD 21702; email; waheedab@mail.nih.gov; Tel. (301) 846-1739; Fax. (301) 846-6777

Keywords: host-pathogen interaction, virus assembly, innate immunity, virus release, lysosome, vacuolar ATPase

\begin{abstract}
The HIV-1 accessory protein Vpu enhances virus release by down-regulating cell surface expression of the host restriction factor tetherin. To further understand the role of host proteins in $\mathrm{Vpu}$ function, we carried out yeast two-hybrid screening and identified the V0 subunit C of vacuolar ATPase (ATP6V0C) as a Vpu-binding protein. To examine the role of ATP6V0C in Vpu-mediated tetherin degradation and HIV-1 release, we knocked down ATP6V0C expression in HeLa cells and observed that ATP6V0C depletion impairs Vpu-mediated tetherin degradation, resulting in a defect in HIV-1 release. We also observed that overexpression of ATP6V0C stabilizes tetherin expression. This stabilization is specific to ATP6V0C, as overexpression of another subunit of the vacuolar ATPase, ATP6V0C", had no effect on tetherin expression. ATP6V0C overexpression did not stabilize CD4, another target of Vpu-mediated degradation. Immunofluorescence localization studies showed that the ATP6V0C-stabilized tetherin is sequestered in a CD63- and LAMP1positive intracellular compartment. These data demonstrate that the Vpu-interacting protein ATP6V0C plays a role in regulating tetherin expression and HIV-1 assembly and release.
\end{abstract}

\section{Introduction}

A number of host proteins possessing antiviral activity have evolved as the first line of defense to suppress the replication of viruses in a cell-autonomous manner. These host proteins, which are often referred to as restriction factors, include APOBEC and SERINC family members, TRIM5 $\alpha$, SAMHD1, and tetherin. Many of these antiviral factors are either expressed constitutively or are induced by type-I interferon (IFN) (in the case of the so-called IFN-stimulated genes, or ISGs) as a component of the innate immune system (1-4). Tetherin [also known as bone marrow stromal antigen-2 (BST-2), cluster of differentiation 317 (CD317) or HM1.24] is an IFN-inducible, type II transmembrane (TM) glycoprotein that interferes with the late stage of the virus replication cycle by tethering virions to the cell surface $(5,6)$. Tetherin was originally identified as a marker for bone marrow stromal cells; it is expressed constitutively in terminally differentiated B cells and $\mathrm{T}$ cells, monocytes, and dendritic cells, and is upregulated in some cancer cells (7-12). Tetherin comprises 180 amino acids and is localized in lipid rafts at the cell surface and on intracellular membranes $(11,13)$. Tetherin has an unusual topology: it contains an $\mathrm{N}$ - 
terminal cytoplasmic tail (CT), an $\alpha$-helical TM domain followed by an extracellular coiled-coil (CC) domain, and a putative Cterminal glycosylphosphatidylinositol (GPI) anchor. Because tetherin has two membrane anchors (TM and GPI-anchor) it is associated with the plasma membrane, specifically in cholesterol- and sphingolipid-rich membrane microdomains (13). The CC ectodomain of human tetherin contains three Cys residues that are essential for the formation of homodimers via disulfide bonding, and two Asn residues that are modified with N-linked oligosaccharides (13-15). Both membrane anchors, and $\mathrm{CT}$ and $\mathrm{CC}$ domains, and the three Cys residues in the $\mathrm{CC}$ domain are essential for the antiviral activity of tetherin $(5,15,16)$. We reported previously that high-mannose modification of a single asparagine residue is necessary and sufficient, whereas complextype glycosylation is dispensable, for cellsurface expression and antiviral activity of tetherin (17). Tetherin inhibits the release of a variety of enveloped viruses including not only HIV-1 but also other lentiviruses, other retroviruses, and alphaviruses, filoviruses, arenaviruses, paramyxoviruses, rhabdoviruses, flaviviruses, orthomyxoviruses, orthohepadnaviruses, and herpesviruses (reviewed in (18-20)).

Several lentiviral proteins have acquired the ability to counteract the antiviral activity of tetherin. The envelope (Env) glycoprotein of HIV-2 and some strains of simian immunodeficiency virus (SIV) counteracts tetherin by sequestering it in a perinuclear compartment, thereby downregulating its expression from the cell surface (21-23). The Nef proteins from SIVcpz and SIVgor antagonize the respective non-human primate tetherin orthologs by decreasing their cell surface expression without inducing their degradation, possibly by intracellular sequestration (24-26). The glycoprotein $\mathrm{M}$ of herpes simplex virus 1 (HSV-1), the Env glycoproteins of equine infectious anemia virus (EIAV), feline immunodeficiency virus (FIV), and HERV-K human endogenous retrovirus, K5 of Kaposi's sarcoma-associated herpesvirus (KSHV), and the non-structural protein 1
(nsP1) of chikungunya virus (CHIKV) antagonize tetherin by distinct mechanisms (27-31). HIV-1 Vpu antagonizes human, chimpanzee, and gorilla tetherin but is relatively inactive against tetherin orthologs from other non-human primates and nonprimate species $(25,32,33)$. Mechanisms by which Vpu antagonizes tetherin include (i) removing it from virus budding sites, (ii) promoting its degradation and/or (iii) downregulating its cell-surface expression.

The accessory protein $\mathrm{Vpu}$ is a $16-\mathrm{kDa}$, 81-amino-acid type I integral membrane phosphoprotein containing a short luminal Nterminal domain, a 23-amino acid TM domain, and a long CT $(34,35)$. The CT of Vpu consists of two $\alpha$-helices linked by a short loop that contains two serine residues (S52 and S56) that undergo phosphorylation. $\mathrm{Vpu}$ is primarily localized in the endoplasmic reticulum (ER), trans-Golgi network (TGN), endosomal membranes, and to some extent at the plasma membrane $(36,37)$. Two main functions have been attributed to Vpu: (i) proteasomal degradation of newly synthesized CD4 receptor (38-40) and (ii) down-regulation of tetherin from virus assembly sites to promote the release of viral particles $(5,6)$. The interaction of Vpu and CD4 via their CTs leads to the recruitment of beta-transducin repeatscontaining protein $(\beta-\mathrm{TrCP})$, followed by $\mathrm{CD} 4$ ubiquitylation and proteasomal degradation (41), whereas interaction between $\mathrm{Vpu}$ and tetherin is mediated by their TM domains (32). Other functions of $\mathrm{Vpu}$ include downregulation of a number of host cell proteins, including major histocompatibility complex class II, tetraspanin family proteins and P-selectin glycoprotein ligand-1, from the cell surface (42-45). Vpu-mediated downregulation of CD4 and tetherin from the cell surface helps to protect HIV-1-infected cells from antibodydependent cell-mediated cytotoxicity (4244,46).

To better understand Vpu function, and identify Vpu-interacting cellular proteins, we previously performed a yeast two-hybrid (Y2H) screen with HIV-1 Vpu as bait. We confirmed that small glutamine-rich 
tetratricopeptide repeat (TPR)-containing protein $\alpha$ (SGTA) is a Vpu-binding protein $(47,48)$. Overexpression of SGTA impaired HIV-1 release independent of Vpu and tetherin expression (47-50), whereas the depletion of SGTA had no significant effect on HIV-1 release or Vpu-mediated tetherin degradation (48). Further, we reported that overexpression of SGTA in the presence of Vpu induced a marked stabilization and cytosolic accumulation of the non-glycosylated form of tetherin. This accumulation of nonglycosylated tetherin in the presence of SGTA was due to inhibition of its degradation, partly due to a block in the translocation of tetherin across the ER membrane resulting in tetherin accumulation in the cytosol (48). The Cterminus of SGTA, the membrane-proximal basic residues of $\mathrm{Vpu}$, and the transmembrane domain of human tetherin are required for SGTA-mediated stabilization of nonglycosylated tetherin (48).

In this study, we report the V0 subunit $\mathrm{C}$ of vacuolar ATPase (ATP6V0C) as another Vpu-interacting protein identified in our $\mathrm{Y} 2 \mathrm{H}$ screen. The vacuolar $\mathrm{H}^{+}$-ATPase (V-ATPase) is a large, membrane-associated, multisubunit enzyme complex that functions as an ATPdependent proton pump that acidifies intracellular compartments such as endosomes, lysosomes, Golgi-derived vesicles, clathrincoated vesicles, synaptic vesicles, multivesicular bodies, and secretory vesicles (reviewed in (51-53)). Acidification of vacuolar compartments plays an important role in several cellular processes such as protein sorting, endocytosis, and macromolecular processing and degradation. The V-ATPase is composed of two domains: a cytoplasmic V1 domain and a transmembrane V0 domain. The V1 domain, which is responsible for ATP hydrolysis, is composed of eight subunits (A$\mathrm{H}$, in a stoichiometry of $\mathrm{A}_{3} \mathrm{~B}_{3} \mathrm{CDEFG}_{2} \mathrm{H}_{1-2}$ ); the V0 subunit, which forms the $\mathrm{H}^{+}$transporting channel, is composed of five subunits $(a, b, c$, c', c" in a stoichiometry of adc 4 c'c" (54-56). ATP hydrolysis catalyzed by subunit V1 drives rotation of the D-F axle, which induces proton translocation across the membrane by rotating the ring of $\mathrm{c}$ subunits. ATP6V0C, a $16-\mathrm{kDa}$ protein with four TM domains (53), has been reported to form gap junctions $(57,58)$, and interacts with a number of cellular and viral proteins independent of other V-ATPase subunits; these include $\beta$-integrin (59), and the bovine papillomavirus E5 oncoprotein $(60,61)$. Knockdown of ATP6V0C induced a synergistic growth-inhibitory effect in the presence of a candidate anti-cancer therapeutic in colorectal cancer cells (62), and attenuated proliferation, invasion, and glucose metabolism in esophageal cancer cells (63). Bafilomycin $\mathrm{A} 1$, an inhibitor of V-ATPase activity, binds ATP6V0C and inhibits proton translocation into the lysosomal lumen, thus inhibiting lysosomal acidification and cargo degradation (64). Inhibition of endosomal acidification by bafilomycin A1 abolished the infection of several viruses such as dengue virus (65), Zika virus (66), human papillomavirus (67), rhinovirus (68), equine infectious anemia virus (69), murine leukemia virus (70), and influenza A virus (71-73).

In this study, we investigated the role of ATP6V0C in Vpu-mediated tetherin degradation and HIV-1 release. Because ATP6V0C is involved in lysosomal degradation, and $\mathrm{Vpu}$ promotes the lysosomal degradation of tetherin, we investigated the effect of ATP6V0C depletion on HIV-1 particle production in the presence and absence of Vpu. We observed that knockdown of ATP6V0C in HeLa cells impairs Vpu-mediated tetherin degradation, thereby inhibiting HIV-1 release. We also observed that overexpression of ATP6V0C resulted in the stabilization of tetherin expression by inducing its sequestration in CD63- and LAMP1-enriched intracellular compartments. Our results demonstrate that the Vpu-interacting protein ATP6V0C plays a role in Vpu-mediated tetherin degradation and HIV-1 release.

\section{Results}

\section{Identification of ATP6V0C as a Vpu- interacting protein}

To identify cellular factors that are involved in Vpu function, we carried out $\mathrm{Y} 2 \mathrm{H}$ screening using full-length $\mathrm{Vpu}$ as bait (for 
details, see experimental procedures). A number of putative Vpu-interacting proteins were identified. Previously, we reported the role of the Vpu-interacting protein SGTA in Vpu-mediated tetherin degradation and HIV-1 release (48). In this study, we investigated the role of another $\mathrm{Vpu}$-interacting protein, ATP6V0C, in Vpu-mediated tetherin degradation and HIV-1 release. First, to confirm the interaction between $\mathrm{Vpu}$ and ATP6V0C identified by $\mathrm{Y} 2 \mathrm{H}$ screening, we carried out co-immunoprecipitation assays. HEK293T cells were transfected with a FLAGtagged ATP6V0C expression vector with or without a Vpu expression vector, and cell lysates were immunoprecipitated with antiFLAG antibodies followed by immunoblotting with anti-FLAG and anti-Vpu antibodies. As shown in Fig. 1A, consistent with the $\mathrm{Y} 2 \mathrm{H}$ result, $\mathrm{Vpu}$ co-immunoprecipitated with ATP6V0C (lane 4, upper right panel). To further confirm this interaction, we performed reciprocal co-immunoprecipitation assays. HEK293T cells were transfected with the Vpu expression vector in the presence and absence of ATP6V0C-expressing plasmid, and cell lysates were immunoprecipitated with anti-Vpu antibodies followed by immunoblotting with anti-Vpu and anti-FLAG antibodies. Again, ATP6V0C co-immunoprecipitated with Vpu (Fig. 1B, lane 4, lower right panel). These coimmunoprecipitation data confirm the $\mathrm{Y} 2 \mathrm{H}$ result and demonstrate the interaction between Vpu and ATP6V0C.

\section{Overexpression of ATP6V0C has no effect on HIV-1 release}

In our previous study, we observed that overexpression of the Vpu-interacting protein SGTA inhibited HIV-1 release in a Vpu- and tetherin-independent manner (48). To investigate whether overexpression of ATP6V0C also inhibits HIV-1 particle release we co-transfected HEK293T cells with a vector expressing FLAG-tagged ATP6V0C in the absence and presence of a tetherin expression vector and WT or Vpu-deleted (delVpu) HIV1 molecular clones. One day posttransfection, cell and viral lysates were subjected to western blot analysis. Overexpression of ATP6V0C had no significant effect on the release of either WT or delVpu HIV-1 (Fig. 2A and B, lanes 1-3 and 7-9) in the absence of tetherin expression. In the presence of tetherin, as expected, the release of delVpu HIV-1 was reduced, but co-expression of ATP6V0C had little or no additional effect on the release of either WT or delVpu HIV-1 (Fig. 2A and B, lanes 4-6 and 10-12). However, we observed that ATP6V0C overexpression in the context of delVpu HIV-1 led to a marked increase in the levels of tetherin expression, in particular a 26-kDa tetherin species (Fig. 2A, lanes 11, and 12). We also observed an increase in tetherin expression in the presence of WT HIV-1 (Fig. 2A, lanes 5 and 6). As expected, in the absence of Vpu (Fig. 2A, lanes 10-12), higher molecular weight tetherin species were observed due to lack of Vpu-mediated tetherin degradation. These results demonstrate that ATP6V0C overexpression markedly increases tetherin levels.

\section{Overexpression of ATP6V0C increases the levels of tetherin but reduces CD4 expression}

In the above experiment, ATP6V0C and tetherin expression vectors were cotransfected with the full-length HIV-1 molecular clone. Next, we tested the expression of tetherin in the presence of ATP6V0C and $\mathrm{Vpu}$ in the absence of other HIV-1 proteins. HEK293T cells were transfected with tetherin expression vectors in the presence and absence of plasmids expressing ATP6V0C and Vpu. As shown in Fig. 3A, overexpressing ATP6V0C markedly increased the abundance of tetherin both in the absence and presence of Vpu. Next, we tested the effect of ATP6V0C overexpression on levels of CD4, a protein that, like tetherin, is downregulated by $\mathrm{Vpu}(40)$. Unlike tetherin, the expression of CD4 was reduced in the presence of ATP6V0C in a dosedependent manner. As expected, co-expression of $\mathrm{Vpu}$ reduced the levels of $\mathrm{CD} 4$, and coexpression of ATP6V0C and Vpu further reduced CD4 levels. These results suggest that two Vpu-downregulated proteins, tetherin and CD4, are regulated differentially by ATP6V0C. As observed earlier (Fig. 1), in this experiment we also observed that $\mathrm{Vpu}$ levels were markedly reduced by ATP6V0C overexpression (Fig. 3B). However, the 
reduction in Vpu levels induced by ATP6V0C overexpression was not seen when tetherin was co-expressed (as seen in Fig. 3A). These results suggest that ATP6V0C down-regulates Vpu expression but co-expression of tetherin prevents Vpu degradation.

We next investigated whether the increase in tetherin expression is specific for the V0C subunit of V-ATPase. To test this, we overexpressed ATP6V0C", another V0 subunit of V-ATPase. In contrast to our observations with ATP6V0C, overexpressing ATP6V0C" modestly increased tetherin expression (3.3fold increase with ATP6V0C" compared to 10.7-fold increase with ATP6V0C) (Fig. 3C). Because V-ATPases are involved in lysosomal degradation, we also examined the effect of overexpressing the V0C and V0C" subunits on the levels of transferrin receptor (TR), which, like tetherin, is a lysosomally degraded protein (74). In contrast to our observations with tetherin, we observed that overexpressing either V0C or V0C" subunits reduced the levels of TR (Fig. 3D). These results suggest that overexpression of V0 subunits of V-ATPase does not globally impair the lysosomal degradation pathway, but rather increases the lysosomal degradation of TR.

\section{Tetherin co-immunoprecipitates with ATP6V0C and this interaction is independent of $\mathrm{Vpu}$}

The data presented in Figs. 2A, 3A, and

$3 \mathrm{C}$ demonstrate that overexpression of the Vpu-interacting protein ATP6V0C increases tetherin expression. Because this phenomenon takes place even in the absence of $\mathrm{Vpu}$, we speculated that ATP6V0C may directly interact with tetherin. To test this hypothesis, we carried out co-immunoprecipitation assays. HEK293T cells were transfected with FLAG-tagged ATP6V0C expression vectors in the absence and presence of $\mathrm{Vpu}$ and HA-tagged tetherin, and immunoprecipitated with anti-FLAG antibodies followed by immunoblotting with anti-FLAG, HA and Vpu antibodies. As shown in Fig 4A, tetherin co-immunoprecipitated with ATP6V0C. As shown in Fig. 1, we also observed $\mathrm{Vpu}$ in the co-immunoprecipitated fraction (Fig. 4A). To confirm these results, we carried out reciprocal co-immunoprecipitation assays. HEK 293T cells were transfected with HA-tagged tetherin in the absence and presence of $\mathrm{Vpu}$ and FLAG-tagged ATP6V0C and immunoprecipitated with anti-HA antibodies followed by immunoblotting with anti-HA, FLAG, and Vpu antibodies. Here again, we could efficiently pull down ATP6V0C both in the absence and presence of Vpu (Fig. 4B). We also observed co-immunoprecipitation of $\mathrm{Vpu}$ with tetherin as reported in the literature $(75,76)$. These results suggest that these three proteins interact independently with one another, i.e., $\mathrm{Vpu}$ interacts with ATP6V0C (Figs. 1 and 4), ATP6V0C interacts with tetherin (Fig.4), and tetherin interacts with Vpu (Fig. 4).

ATP6V0C overexpression stabilizes tetherin

To elucidate the mechanism by which ATP6V0C increases the levels of tetherin, we performed pulse-chase analysis. 293T cells expressing tetherin alone or coexpressing tetherin with ATP6V0C were pulse-labeled with $\left[{ }^{35} \mathrm{~S}\right] \mathrm{Met} / \mathrm{Cys}$ for $30 \mathrm{~min}$ and chased for 0 , $0.5,1,2,4 \mathrm{~h}$ in unlabeled medium. We observed that ATP6V0C overexpression increased the half-life of tetherin (Fig. 5). Quantification indicated that the half-life of the 23- and 26-kDa tetherin species was 32 and 120 min in the absence and presence of ATP6V0C overexpression, respectively. These results indicate that ATP6V0C overexpression prevents the degradation of tetherin, leading to its stabilization and time-dependent accumulation.

The cytoplasmic tail, GPI-anchor, and dimerization of human tetherin are required, whereas glycosylation and the transmembrane domain are dispensable, for ATP6V0C-mediated tetherin stabilization

We next used several tetherin mutants to investigate which domain(s) of tetherin are essential for ATP6V0C-mediated tetherin stabilization. As shown in Fig. 6A, the expression of the non-glycosylated double mutant $(\mathrm{N} 65,92 \mathrm{~A})$ is increased, whereas the non-dimerizing (CCC), cytoplasmic taildeleted (delCT), and GPI-anchor-deleted (delGPI) mutants show no increased expression upon ATP6V0C overexpression (Fig. 6A). We 
also tested tetherin proteins from other species, African green monkey (Agm) and rhesus macaque, that are not downregulated by Vpu due lack of Vpu-tetherin interaction. Unlike human tetherin, Agm and rhesus tetherins were not stabilized. Chimeras in which the transmembrane domain of human tetherin was replaced with the corresponding sequences from Agm or rhesus tetherin (Hu-Agm and $\mathrm{Hu}-$ $\mathrm{Rh}$, respectively) were also stabilized by ATP6V0C overexpression. These results indicate that the transmembrane domain and glycosylation of human tetherin are dispensable whereas the cytoplasmic tail, GPIanchor, and dimerization of tetherin are required for ATP6V0C-mediated stabilization.

\section{Stabilization of tetherin is not due to inhibition of lysosomal degradation}

The results presented above (Figs. 3A, $\mathrm{B}$ and D) demonstrate that although ATP6V0C overexpression stabilizes tetherin expression, it reduces the levels of two lysosomally degraded proteins, CD4 and TR. To explore further the connection between ATP6V0C overexpression and lysosomal function, we treated cells with bafilomycin, which specifically binds the ATP6V0C subunit and prevents proton transport and lysosomal acidification (77). We observed an increase in the expression of tetherin in bafilomycin-treated cells (Fig. 7A). Strikingly, the expression of tetherin is much higher in ATP6V0C overexpressing cells that are treated with bafilomycin relative to cells not treated with the lysosomal inhibitor. The higher expression of tetherin in ATP6V0C-expressing cells treated with bafilomycin could be due to the additive effect of tetherin stabilization by ATP6V0C and inhibition of degradation by bafilomycin. Next, we tested the effect of overexpressing ATP6V0C on Vpu expression. As shown in Fig. 7B, the expression of Vpu is reduced by co-expressing ATP6V0C. These results confirm our earlier observation in Fig. $3 \mathrm{~B}$, which was made in the presence of $\mathrm{CD} 4$ (Fig. 3B). Treating these cells with bafilomycin increases the expression of $\mathrm{Vpu}$, suggesting that ATP6V0C overexpression leads to degradation of $\mathrm{Vpu}$ through the lysosomal pathway (Fig. 6B). This reduction in the expression of $\mathrm{Vpu}$ induced by ATP6V0C overexpression is not observed in the presence of tetherin (Fig. 2A lanes 5 and 6, and Fig. 3A, lanes 5 and 6$)$. These observations suggest that tetherin binding to ATP6V0C reduces the ability of ATP6V0C to down-regulate Vpu, consistent with our finding that ATP6V0C, $\mathrm{Vpu}$, and tetherin form a trimeric complex.

\section{ATP6V0C overexpression sequesters tetherin in lysosomal and CD63-positive compartments}

To investigate the effect of ATP6V0C overexpression on tetherin localization, we performed immunofluorescence microscopy analysis. 293T cells were transfected with HAtetherin in the absence and presence of $\mathrm{C}$ terminally FLAG-tagged ATP6V0C, and cells were fixed and stained with anti-HA and antiFLAG antibodies. As reported previously (13) tetherin is normally localized predominantly on the cell surface (Fig. 8A). Upon ATP6V0C overexpression, the localization of tetherin markedly shifts from the plasma membrane to intracellular vesicular compartments. Interestingly, ATP6V0C is also localized in these compartments, resulting in high colocalization of tetherin and ATP6V0C [Pearson correlation coefficient (R) value $0.81 \pm 0.09$ (Fig. 8A)]. We next characterized the compartments in which tetherin is sequestered by ATP6V0C. 293T cells expressing tetherin in the absence and presence of ATP6V0C were stained for tetherin and the trans-Golgi network (TGN) marker TNG46, the late endosome marker CD63, or lysosomal marker LAMP-1. We observed little or no colocalization between tetherin and TNG46 (data not shown); however, we observed high levels of co-localization of tetherin with both CD63 [(Fig. 8B), $\mathrm{R}=0.757 \pm 0.088$ ] and LAMP1 [(Fig. 8C) $\mathrm{R}=0.758 \pm 0.089$ ]. These results indicate that ATP6V0C sequesters tetherin in a compartment that is positive for late endosomal and lysosomal markers. That ATP6V0C induces accumulation of tetherin in CD63- and LAMP-1-positive compartments without inducing tetherin degradation suggests that the late endosomal and LAMP-1-positive vesicles in which tetherin accumulates represent an aberrant, non-functional lysosomal compartment. 
We next overexpressed individual tetherin mutants in the presence of ATP6V0C and examined the localization of tetherin and ATP6V0C. CT-deleted tetherin and the nondimerizing tetherin mutant, which are both localized to a perinuclear region, are not sequestered by ATP6V0C (Fig. S1A and S1B). Interestingly, in the presence of these tetherin mutants, ATP6V0C itself is diffusely localized in the cytosol. The GPI-deleted tetherin mutant, which is localized on the plasma and internal membranes, is also not sequestered by ATP6V0C (Fig. S1C) and ATP6V0C localization remains cytosolic. However, the non-glycosylated tetherin mutant is sequestered and colocalizes with ATP6V0C in an internal compartment (Fig. S1D). These results correlate with our biochemical observations in Fig. 6A; i.e. tetherin molecules that are stabilized by ATP6V0C overexpression colocalize with ATP6V0C in an internal compartment, putatively an aberrant late endosome or lysosome.

\section{Knockdown of ATP6V0C inhibits HIV-1 release by enhancing tetherin expression}

To investigate the role of endogenous ATP6V0C in tetherin expression and HIV-1 release, we knocked-down ATP6V0C expression in HeLa cells with siRNA (knockdown efficiency $>90 \%$; Fig. 9C) and analyzed the expression of tetherin and HIV-1 release. Knocking-down ATP6V0C increased the expression of endogenous tetherin, resulting in a reduction in WT virus release efficiency (Fig. 9A and B). As expected (78,79), in the absence of ATP6V0C knock-down, the release of delVpu HIV-1 is reduced in HeLa cells because of the absence of Vpu-mediated tetherin degradation (Fig. 9A and B). Knock-down of ATP6V0C increased tetherin expression and further reduced delVpu release. Knocking down tetherin along with ATP6V0C rescued the release of both WT and delVpu HIV-1, suggesting that the impaired virus release observed upon knocking down ATP6V0C is due to higher tetherin expression. These results demonstrate that ATP6V0C is required for both normal turnover of tetherin and Vpu-mediated tetherin degradation in HeLa cells. Further, to confirm that the reduction in virus release observed upon ATP6V0C knock-down in HeLa cells is due to increased levels of tetherin, we knocked-down ATP6V0C in 293T cells, which do not express tetherin. In $293 \mathrm{~T}$ cells, we do not see any significant reduction in virus release upon knock-down of ATP6V0C (Fig 9D and $\mathrm{E}$ ), consistent with the hypothesis that the decrease in HIV-1 release in ATP6V0Cdepleted HeLa cells is due to increased tetherin expression. Interestingly, the levels of TR are not reduced by knock-down of ATP6V0C (Fig. 9D).

\section{Discussion}

We identified SGTA and ATP6V0C as Vpu-interacting proteins in a $\mathrm{Y} 2 \mathrm{H}$ screen. In this study, we confirmed the interaction between $\mathrm{Vpu}$ and ATP6V0C using coimmunoprecipitation assays. Although in our earlier study siRNA-mediated depletion of SGTA had no significant effect on the release of either WT or Vpu-defective HIV-1 in tetherin-expressing HeLa cells, overexpression of SGTA interfered with HIV-1 release in a Vpu- and tetherin-independent manner (48). In this study, we observe that overexpression of ATP6V0C has no significant effect on HIV-1 release, whereas knockdown of ATP6V0C in HeLa cells, but not in tetherin-negative 293T cells, inhibits the release of both $\mathrm{Vpu}(+)$ and Vpu(-) HIV-1. The inhibition of virus release mediated by ATP6V0C depletion in HeLa cells is rescued by knockdown of endogenous tetherin, indicating that the inhibition of HIV-1 release upon knockdown of ATP6V0C is mediated by tetherin. Further, we observe that ATP6V0C depletion in HeLa cells results in elevated levels of endogenous tetherin independent of Vpu expression, indicating that endogenous ATP6V0C is not only required for Vpu-mediated tetherin degradation but also for normal tetherin turnover. We and others have reported that $\mathrm{Vpu}$ induces the degradation of tetherin by both proteasomal (80-83) and lysosomal $(75,83,84)$ pathways. Because ATP6V0C regulates vesicular acidification required for lysosomal degradation (51-53), we suggest that ATPV0C is essential for Vpu- 
mediated lysosomal tetherin degradation to promote HIV-1 release.

We observed that ATP6V0C overexpression results in the accumulation of tetherin by preventing its degradation and that this accumulation is Vpu-independent. Overexpression of another subunit of the VATPase, ATP6V0C", has only a small effect on tetherin expression compared to ATP6V0C, indicating that tetherin stabilization is specific to ATP6V0C. Further, the ATP6V0C-mediated stabilization is specific to tetherin, as overexpression of ATP6V0C did not stabilize the Vpu-downregulated protein CD4 or interfere with its Vpu-mediated downregulation. Overexpression of ATP6V0C reduced the expression of CD4 and TR, both of which undergo lysosomal degradation (38-40). Treating ATP6V0C overexpressing cells with the lysosomal inhibitor bafilomycin, which specifically targets the ATP6V0C subunit and prevents lysosomal degradation (64), further increased tetherin expression. Overexpression of ATP6V0C reduced the expression of Vpu, which could be rescued by treating cells with bafilomycin, indicating that ATP6V0C overexpression leads to degradation of $\mathrm{Vpu}$ through the lysosomal pathway. These results indicate that overexpression of ATP6V0C does not exert a dominant-negative effect on lysosomal function. Our coimmunoprecipitation assays demonstrate that Vpu, ATP6V0C, and tetherin interact independently of one another. Together, these results suggest that ATP6V0C interaction with tetherin prevents its degradation, leading to its accumulation. Using deletion and point mutants, we determined that the cytoplasmic tail, GPI-anchor, and dimerization of tetherin are essential for ATP6V0C6-mediated tetherin stabilization. Immunofluorescence localization studies showed that the ATP6V0C-stabilized tetherin is sequestered in CD63- and LAMP1enriched intracellular compartments, whereas tetherin mutants that are not stabilized by ATP6V0C are not sequestered in these internal compartments. The observation that ATP6V0C increases tetherin levels without affecting HIV-1 release is explained by the intracellular sequestration of ATP6V0C- stabilized tetherin; this stabilized tetherin is not available to inhibit particle release at the plasma membrane.

The basis for the interaction between $\mathrm{Vpu}$ and ATP6V0C remains unclear. Vpu could have evolved an interaction with ATP6V0C to antagonize the activity of the VATPase or to promote its activity in degrading Vpu-target proteins. Because ATP6V0C is a pore-forming and proton-conducting subunit of the V-ATPase, we initially anticipated that the interaction of $\mathrm{Vpu}$ with overexpressed ATP6V0C might facilitate Vpu-mediated tetherin degradation. However, we observed that overexpression of ATP6V0C resulted in the accumulation, rather than lysosomal degradation, of tetherin. Although overexpression of ATP6V0C increases the levels of tetherin, this effect is independent of $\mathrm{Vpu}$. Interactions between virally encoded factors and ATP6V0C or other V-ATPase subunits have been reported in other systems. For example, a direct interaction between bovine and human papillomavirus protein E5 and ATP6V0C has been reported $(60,85)$. Although several studies have demonstrated that E5 disrupts acidification of endosomes (86-88) this activity appears to be independent of E5-ATP6V0C binding (89). A human cytomegalovirus (hCMV) microRNA has been found to target ATP6V0C (90), which, paradoxically, is required for efficient hCMV virus assembly in culture (91). Why hCMV would target for degradation a host protein required for its replication has not been elucidated. The non-structural protein $3 \mathrm{~A}$ of enterovirus 71 (EV71) interacts with ATP6V0C and this interaction is critical for EV71 replication, as knockdown of ATP6V0C or treating cells with bafilomycin inhibits propagation of EV71 (92). Dengue virus premembrane (prM) protein interacts with VATPase and this interaction is essential for both entry and egress of dengue virus (93). HIV-1 and SIV Nef have been reported to interact with the $\mathrm{H}$ subunit of the V-ATPase, thereby connecting Nef and the endocytosis machinery required for Nef-mediated CD4 downregulation (94-96). Finally, HTLV-1 p12I has been reported to interact with ATP6V0C 
$(97,98)$; the implications of this interaction remain to be defined.

In the course of this study, we also observed that the Vpu target protein, tetherin, interacts with ATP6V0C. As a consequence of this interaction, overexpression of ATP6V0C leads to the stabilization of tetherin and the sequestration of tetherin in an internal compartment that contains CD63 and LAMP-1. Variants of tetherin that are not stabilized by ATP6V0C fail to relocalize to the internal compartment. Whereas ATP6V0C overexpression stabilizes tetherin, it causes a reduction in the levels of TR and CD4, two proteins that undergo lysosomal degradation. These results suggest that the stabilization of tetherin by ATP6V0C overexpression is a result of the (direct or indirect) interaction between tetherin and ATP6V0C and is not a consequence of global disruption of lysosomal activity.

In summary, in this report, we show that overexpressed ATP6V0C interacts with tetherin and stabilizes its expression by preventing study-state degradation and sequestration in intracellular compartments. In contrast, endogenous ATP6V0C is required for Vpu-mediated tetherin degradation and enhancement of HIV-1 release in HeLa cells. These results demonstrate that the $\mathrm{Vpu}-$ interacting protein ATP6V0C regulates the expression of tetherin and HIV-1 release.

\section{Experimental procedures}

\section{Plasmids, antibodies, and chemicals}

Vectors expressing C-terminally FLAG-tagged ATP6V0C and ATP6V0C" were obtained from OriGene Technologies Inc. (Rockville, MD). The pcDNA-Vphu vector, bearing the codon-optimized $v p u$ gene, was used for expressing Vpu (99). The full-length, infectious HIV-1 molecular clone pNL4-3 and the Vpu-defective counterpart pNL4-3delVpu were described previously $(35,100)$. pNL43 delVpu and pcDNA-Vphu were kindly provided by K. Strebel. Vectors expressing Nterminally HA-tagged human tetherin, the deletion mutant derivatives delCT and delGPI, tetherin from African green monkey (Agm) and Rhesus ( $\mathrm{Rh}$ ), and chimeric human tetherin with transmembrane domains from Agm (Hu-Agm) or $\mathrm{Rh}(\mathrm{Hu}-\mathrm{Rh})$ were described previously $(5,32)$, and were generously provided by $\mathrm{P}$. Bieniasz. The non-glycosylated (NN) and nondimerizing (CCC) tetherin mutants have been described previously (48). The CD4 expression vector $\mathrm{pMX}-\mathrm{CD} 4$ and the TR expression vector pMD18-T TR were from Addgene (Watertown, MA) and Sino Biological Inc. (Wayne, PA), respectively. Anti-FLAG, anti-HA, and antitubulin antibodies, and anti-HA- and antiFLAG-conjugated agarose beads were purchased from Sigma (St. Louis, MO). The following siRNAs targeting human genes were obtained: ATPV0C from Sant Cruz Biotechnology (Dallas, Texas), ATP6V0C" from OriGene Technologies Inc., tetherin from Qiagen (Germantown, MD), and non-targeting control-siRNA from Dharmacon Inc. (Chicago, IL, now part of Horizon Discovery). Antihuman TR antibody was purchased from Zymed Laboratories Inc. (San Francisco, CA), anti-CD4 and anti-CD63 antibodies were from Santa Cruz, and anti-LAMP-1 from BD Biosciences (San Jose, CA). Bafilomycin was obtained from Tocris Bioscience (Minneapolis, $\mathrm{MN}$ ). Zenon antibody labeling kits and the Alexa Fluor 488, 594, and 647 conjugated secondary antibodies were from Invitrogen (Grand Island, NY). Anti-Vpu (34), anti-BST2 (101), and anti-HIV-1 Ig were obtained from the NIH AIDS Reagent Program.

\section{Yeast two-hybrid screening}

The yeast two-hybrid screen was carried out at Myriad Genetics (Salt Lake City, UT) as described previously (48). Briefly, the PNY200 yeast strain expressing the Vpu-fused GAL4 DNA binding protein was mated with the BK100 yeast strain transformed with a prey cDNA library (isolated from brain, spleen, and macrophages). After mating, the cells were plated onto selective media and the positive colonies were picked from the selection plates (102). The prey plasmids were isolated from the positive colonies, and the prey inserts were identified by sequence analysis. The interactions were confirmed by transforming the bait and prey plasmids into naïve yeast cells 
and monitoring the interactions by the chemiluminescent reporter gene assay system as described previously (102).

\section{Cell culture and transfection}

$293 \mathrm{~T}$ and HeLa cell lines were maintained in Dulbecco-modified Eagle's medium (DMEM) containing $10 \%$ or $5 \%$ fetal bovine serum (FBS), respectively. One day after plating, cells were transfected with appropriate plasmid DNA using Lipofectamine 2000 (Invitrogen Corp., Carlsbad, CA) according to the manufacturer's recommendations. Twenty-four $h$ posttransfection, virions were pelleted in an ultracentrifuge and cell and virus pellets were lysed and used for further analysis. In bafilomycin treatment experiments, $8 \mathrm{~h}$ posttransfection, cells were either untreated or treated with bafilomycin for $18 \mathrm{~h}$ prior to cell lysis.

\section{Immunoprecipitation and western blotting}

For co-immunoprecipitation assays, 293 T cells were transfected with FLAG-tagged ATP6V0C in the absence and presence of vectors expressing $\mathrm{Vpu}$ and human tetherin. After 24 to $30 \mathrm{~h}$ post-transfection, cells were lysed in $0.5 \%$ IGEPAL and immunoprecipitations were performed with agarose beads conjugated with anti-FLAG, anti-Vpu, or anti-HA antibodies. After overnight incubation, complexes were washed with $0.1 \%$ IGEPAL, and both cell lysates and immunoprecipitated samples were subjected to immunoblotting with anti-FLAG, anti-Vpu, and anti-HA antibodies. For immunoblot analyses, cell and virus pellets were lysed in a buffer containing $50 \mathrm{mM}$ Tris- $\mathrm{HCl}(\mathrm{pH} \mathrm{7.4)}$, $150 \mathrm{mM} \mathrm{NaCl}, 1 \mathrm{mM}$ EDTA, $0.5 \%$ Triton X100 , and protease inhibitor cocktail (Roche life sciences, Basel, Switzerland). Proteins were denatured by boiling in sample buffer and subjected to SDS-PAGE, transferred to PVDF membrane, blocked with 5\% milk and incubated with appropriate antibodies as described in the text. Membranes were then incubated with HRP-conjugated secondary antibodies, and chemiluminescence signal was detected by using West Pico or West Femto Chemiluminescence Reagent (Thermo
Scientific, Waltham, MA). The protein bands were quantified by using Imagelab-Chemidoc (Bio-rad Laboratories, France).

\section{Virus release assays}

One day after plating, 293T cells were transfected with WT or Vpu-defective pNL4-3 molecular clones in the absence and presence of ATP6V0C and tetherin expression vectors. One-day posttransfection, virions were pelleted in an ultracentrifuge and cell and virus pellets were lysed (103). To knock down ATP6V0C in HeLa and 293T cells, and tetherin in HeLa cells, one day after plating, cells were transfected with $50 \mathrm{nM}$ non-target or ATP6V0C or tetherin siRNA with Oligofectamine transfection reagent (Invitrogen) in serum-free DMEM. After 6 to 7 $\mathrm{h}$, DMEM containing $15 \%$ FBS was added and cells were cultured overnight. The next day, cells were infected with VSVG-pseudotyped wild-type (WT) or Vpu-defective HIV-1; after $8 \mathrm{~h}$ the medium was changed, and the siRNA transfection was repeated. Two days later, virions were pelleted in an ultracentrifuge and cell and viral pellets were lysed as above. Viral proteins in cell and virus lysates were immunoblotted with HIV-Ig (48) and virus release efficiency was calculated as the amount of virion-associated p24 as a fraction of total (cell-associated p24 and Pr55 plus virionassociated p24) Gag.

\section{Pulse-chase analysis}

293T cells were transfected with HAtagged human tetherin in the absence and presence of ATP6V0C expression vector. One day posttransfection, cells were pulse-labeled with $\left[{ }^{35} \mathrm{~S}\right]$ Met-Cys for $30 \mathrm{~min}$ and washed with DMEM containing 10\% FBS and removed from the dish in the same medium. Cells were split into five equal parts and incubated for 0 , $0.5,1,2,4 \mathrm{~h}$. Cells were spun down after the indicated incubation time and lysed in $0.5 \%$ IGEPAL-containing lysis buffer. The cell lysates were subjected to immunoprecipitation with anti-HA antibody-tagged beads overnight, washed and analyzed by SDS-PAGE followed by fluorography (104).

\section{Immunofluorescence microscopy}


For microscopy studies, $293 \mathrm{~T}$ cells were cultured in chamber slides. One-day after plating, cells were transfected with human tetherin expression vector in the absence or presence of ATP6V0C expression vector. After $24 \mathrm{~h}$, cells were rinsed with PBS and fixed with $3.7 \%$ paraformaldehyde in PBS for $30 \mathrm{~min}$. The cells were rinsed with PBS three times, permeabilized with methanol at $20^{\circ} \mathrm{C}$ for 4 min, washed in PBS and incubated with 0.1 $\mathrm{M}$ glycine-PBS for $10 \mathrm{~min}$ to quench the remaining aldehyde residues. After blocking with 3\% BSA-PBS for $30 \mathrm{~min}$, cells were incubated with anti-HA and anti-FLAG antibodies diluted in 3\% BSA-PBS for $1 \mathrm{~h}$. The cells were washed with PBS three times and then incubated with secondary antibody conjugated with Alexa Fluor 488 and Alexa Fluor 594 diluted in 3\% BSA-PBS for $1 \mathrm{~h}$ to label anti-HA and anti-FLAG, respectively. In triple antibody staining experiments, cells were first incubated with anti-FLAG antibody diluted in 3\% BSA-PBS for $1 \mathrm{~h}$, then cells were washed with PBS three times and then incubated with secondary antibody conjugated with Alexa Fluor 647 diluted in 3\% BSA-PBS. After washing with PBS three times, cells were incubated with Zenon Alexa Fluor 488-labeled anti-HA and Zenon Alexa Fluor 594-labeled anti-CD63 or anti-LAMP-1 for 1h. Finally, after washing with PBS three times, cells were mounted with Vectashield mounting media with DAPI (Vector Laboratories) and examined with a Delta-Vision RT microscope. 


\section{Acknowledgements}

We thank Myriad Genetics (Salt Lake City, UT) for carrying out yeast two-hybrid screening. We thank members of the Freed laboratory for helpful discussion and critical review of the manuscript. We thank K. Strebel and P. Bieniasz for their generous gifts of plasmids. The HIV-1 Ig, anti-Vpu and anti-human tetherin antisera were obtained from the NIH AIDS Reagent Program. Research in the Freed laboratory is supported by the Intramural Research Program of the Center for Cancer Research, National Cancer Institute, NIH.

\section{Author contributions}

A.W. planned, designed and performed the experiments. M.S., A.K., A.G., and A.M. helped in biochemical analyses. E.O.F. coordinated and supervised the project. A.W. and E.O.F. wrote the manuscript.

\section{Conflict of interest}

The authors declare no conflict of interest. 


\section{References}

1. Colomer-Lluch, M., Ruiz, A., Moris, A., and Prado, J. G. (2018) Restriction Factors: From Intrinsic Viral Restriction to Shaping Cellular Immunity Against HIV-1. Front Immunol 9, 2876

2. $\quad$ Forlani, G., Shallak, M., Ramia, E., Tedeschi, A., and Accolla, R. S. (2019) Restriction factors in human retrovirus infections and the unprecedented case of CIITA as link of intrinsic and adaptive immunity against HTLV-1. Retrovirology 16, 34

3. Jia, X., Zhao, Q., and Xiong, Y. (2015) HIV suppression by host restriction factors and viral immune evasion. Curr Opin Struct Biol 31, 106-114

4. V, D. U., De Crignis, E., and Re, M. C. (2018) Host Restriction Factors and Human Immunodeficiency Virus (HIV-1): A Dynamic Interplay Involving All Phases of the Viral Life Cycle. Curr HIV Res 16, 184-207

5. Neil, S. J., Zang, T., and Bieniasz, P. D. (2008) Tetherin inhibits retrovirus release and is antagonized by HIV-1 Vpu. Nature 451, 425-430

6. Van Damme, N., Goff, D., Katsura, C., Jorgenson, R. L., Mitchell, R., Johnson, M. C., Stephens, E. B., and Guatelli, J. (2008) The interferon-induced protein BST-2 restricts HIV-1 release and is downregulated from the cell surface by the viral Vpu protein. Cell host \& microbe 3, 245-252

7. Cai, D., Cao, J., Li, Z., Zheng, X., Yao, Y., Li, W., and Yuan, Z. (2009) Up-regulation of bone marrow stromal protein 2 (BST2) in breast cancer with bone metastasis. BMC Cancer 9, 102

8. Erikson, E., Adam, T., Schmidt, S., Lehmann-Koch, J., Over, B., Goffinet, C., Harter, C., Bekeredjian-Ding, I., Sertel, S., Lasitschka, F., and Keppler, O. T. (2011) In vivo expression profile of the antiviral restriction factor and tumor-targeting antigen CD317/BST-2/HM1.24/tetherin in humans. Proc Natl Acad Sci U S A 108, 13688-13693

9. Goto, T., Kennel, S. J., Abe, M., Takishita, M., Kosaka, M., Solomon, A., and Saito, S. (1994) A novel membrane antigen selectively expressed on terminally differentiated human B cells. Blood 84, 1922-1930

10. Grutzmann, R., Boriss, H., Ammerpohl, O., Luttges, J., Kalthoff, H., Schackert, H. K., Kloppel, G., Saeger, H. D., and Pilarsky, C. (2005) Meta-analysis of microarray data on pancreatic cancer defines a set of commonly dysregulated genes. Oncogene 24, 50795088

11. Ishikawa, J., Kaisho, T., Tomizawa, H., Lee, B. O., Kobune, Y., Inazawa, J., Oritani, K., Itoh, M., Ochi, T., Ishihara, K., and et al. (1995) Molecular cloning and chromosomal mapping of a bone marrow stromal cell surface gene, BST2, that may be involved in preB-cell growth. Genomics 26, 527-534

12. Walter-Yohrling, J., Cao, X., Callahan, M., Weber, W., Morgenbesser, S., Madden, S. L., Wang, C., and Teicher, B. A. (2003) Identification of genes expressed in malignant cells that promote invasion. Cancer Res 63, 8939-8947

13. Kupzig, S., Korolchuk, V., Rollason, R., Sugden, A., Wilde, A., and Banting, G. (2003) Bst-2/HM1.24 is a raft-associated apical membrane protein with an unusual topology. Traffic 4, 694-709

14. Ohtomo, T., Sugamata, Y., Ozaki, Y., Ono, K., Yoshimura, Y., Kawai, S., Koishihara, Y., Ozaki, S., Kosaka, M., Hirano, T., and Tsuchiya, M. (1999) Molecular cloning and characterization of a surface antigen preferentially overexpressed on multiple myeloma cells. Biochem Biophys Res Commun 258, 583-591

15. Perez-Caballero, D., Zang, T., Ebrahimi, A., McNatt, M. W., Gregory, D. A., Johnson, M. C., and Bieniasz, P. D. (2009) Tetherin inhibits HIV-1 release by directly tethering virions to cells. Cell 139, 499-511 
16. Andrew, A. J., Miyagi, E., Kao, S., and Strebel, K. (2009) The formation of cysteinelinked dimers of BST-2/tetherin is important for inhibition of HIV-1 virus release but not for sensitivity to Vpu. Retrovirology 6, 80

17. Waheed, A. A., Gitzen, A., Swiderski, M., and Freed, E. O. (2018) High-Mannose But Not Complex-Type Glycosylation of Tetherin Is Required for Restriction of HIV-1 Release. Viruses $\mathbf{1 0}$

18. Mahauad-Fernandez, W. D., and Okeoma, C. M. (2016) The role of BST-2/Tetherin in host protection and disease manifestation. Immun Inflamm Dis 4, 4-23

19. Neil, S. J. (2013) The antiviral activities of tetherin. Curr Top Microbiol Immunol 371, 67-104

20. Sauter, D. (2014) Counteraction of the multifunctional restriction factor tetherin. Front Microbiol 5, 163

21. Gupta, R. K., Mlcochova, P., Pelchen-Matthews, A., Petit, S. J., Mattiuzzo, G., Pillay, D., Takeuchi, Y., Marsh, M., and Towers, G. J. (2009) Simian immunodeficiency virus envelope glycoprotein counteracts tetherin/BST-2/CD317 by intracellular sequestration. Proc Natl Acad Sci U S A 106, 20889-20894

22. Hauser, H., Lopez, L. A., Yang, S. J., Oldenburg, J. E., Exline, C. M., Guatelli, J. C., and Cannon, P. M. (2010) HIV-1 Vpu and HIV-2 Env counteract BST-2/tetherin by sequestration in a perinuclear compartment. Retrovirology 7,51

23. Le Tortorec, A., and Neil, S. J. (2009) Antagonism to and intracellular sequestration of human tetherin by the human immunodeficiency virus type 2 envelope glycoprotein. $J$ Virol 83, 11966-11978

24. Gotz, N., Sauter, D., Usmani, S. M., Fritz, J. V., Goffinet, C., Heigele, A., Geyer, M., Bibollet-Ruche, F., Learn, G. H., Fackler, O. T., Hahn, B. H., and Kirchhoff, F. (2012) Reacquisition of Nef-mediated tetherin antagonism in a single in vivo passage of HIV-1 through its original chimpanzee host. Cell host \& microbe 12, 373-380

25. Jia, B., Serra-Moreno, R., Neidermyer, W., Rahmberg, A., Mackey, J., Fofana, I. B., Johnson, W. E., Westmoreland, S., and Evans, D. T. (2009) Species-specific activity of SIV Nef and HIV-1 Vpu in overcoming restriction by tetherin/BST2. PLoS Pathog $\mathbf{5}$, e1000429

26. Zhang, F., Wilson, S. J., Landford, W. C., Virgen, B., Gregory, D., Johnson, M. C., Munch, J., Kirchhoff, F., Bieniasz, P. D., and Hatziioannou, T. (2009) Nef proteins from simian immunodeficiency viruses are tetherin antagonists. Cell host \& microbe 6, 54-67

27. Blondeau, C., Pelchen-Matthews, A., Mlcochova, P., Marsh, M., Milne, R. S., and Towers, G. J. (2013) Tetherin restricts herpes simplex virus 1 and is antagonized by glycoprotein M. J Virol 87, 13124-13133

28. Jones, P. H., Maric, M., Madison, M. N., Maury, W., Roller, R. J., and Okeoma, C. M. (2013) BST-2/tetherin-mediated restriction of chikungunya (CHIKV) VLP budding is counteracted by CHIKV non-structural protein 1 (nsP1). Virology 438, 37-49

29. Lemaitre, C., Harper, F., Pierron, G., Heidmann, T., and Dewannieux, M. (2014) The HERV-K human endogenous retrovirus envelope protein antagonizes Tetherin antiviral activity. J Virol 88, 13626-13637

30. Morrison, J. H., Guevara, R. B., Marcano, A. C., Saenz, D. T., Fadel, H. J., Rogstad, D. K., and Poeschla, E. M. (2014) Feline immunodeficiency virus envelope glycoproteins antagonize tetherin through a distinctive mechanism that requires virion incorporation. $J$ Virol 88, 3255-3272

31. Yin, X., Hu, Z., Gu, Q., Wu, X., Zheng, Y. H., Wei, P., and Wang, X. (2014) Equine tetherin blocks retrovirus release and its activity is antagonized by equine infectious anemia virus envelope protein. $J$ Virol 88, 1259-1270 
32. McNatt, M. W., Zang, T., Hatziioannou, T., Bartlett, M., Fofana, I. B., Johnson, W. E., Neil, S. J., and Bieniasz, P. D. (2009) Species-specific activity of HIV-1 Vpu and positive selection of tetherin transmembrane domain variants. PLoS Pathog 5, e1000300

33. Sauter, D., Schindler, M., Specht, A., Landford, W. N., Munch, J., Kim, K. A., Votteler, J., Schubert, U., Bibollet-Ruche, F., Keele, B. F., Takehisa, J., Ogando, Y., Ochsenbauer, C., Kappes, J. C., Ayouba, A., Peeters, M., Learn, G. H., Shaw, G., Sharp, P. M., Bieniasz, P., Hahn, B. H., Hatziioannou, T., and Kirchhoff, F. (2009) Tetherin-driven adaptation of Vpu and Nef function and the evolution of pandemic and nonpandemic HIV-1 strains. Cell host \& microbe 6, 409-421

34. Maldarelli, F., Chen, M. Y., Willey, R. L., and Strebel, K. (1993) Human immunodeficiency virus type $1 \mathrm{Vpu}$ protein is an oligomeric type I integral membrane protein. J Virol 67, 5056-5061

35. Strebel, K., Klimkait, T., and Martin, M. A. (1988) A novel gene of HIV-1, vpu, and its 16-kilodalton product. Science 241, 1221-1223

36. Pacyniak, E., Gomez, M. L., Gomez, L. M., Mulcahy, E. R., Jackson, M., Hout, D. R., Wisdom, B. J., and Stephens, E. B. (2005) Identification of a region within the cytoplasmic domain of the subtype B Vpu protein of human immunodeficiency virus type 1 (HIV-1) that is responsible for retention in the golgi complex and its absence in the $\mathrm{Vpu}$ protein from a subtype C HIV-1. AIDS research and human retroviruses 21, 379-394

37. Varthakavi, V., Smith, R. M., Martin, K. L., Derdowski, A., Lapierre, L. A., Goldenring, J. R., and Spearman, P. (2006) The pericentriolar recycling endosome plays a key role in Vpu-mediated enhancement of HIV-1 particle release. Traffic 7, 298-307

38. Chen, M. Y., Maldarelli, F., Karczewski, M. K., Willey, R. L., and Strebel, K. (1993) Human immunodeficiency virus type 1 Vpu protein induces degradation of CD4 in vitro: the cytoplasmic domain of CD4 contributes to Vpu sensitivity. J Virol 67, 3877-3884

39. Schubert, U., Anton, L. C., Bacik, I., Cox, J. H., Bour, S., Bennink, J. R., Orlowski, M., Strebel, K., and Yewdell, J. W. (1998) CD4 glycoprotein degradation induced by human immunodeficiency virus type $1 \mathrm{Vpu}$ protein requires the function of proteasomes and the ubiquitin-conjugating pathway. $J$ Virol 72, 2280-2288

40. Willey, R. L., Maldarelli, F., Martin, M. A., and Strebel, K. (1992) Human immunodeficiency virus type $1 \mathrm{Vpu}$ protein induces rapid degradation of CD4. J Virol 66, 7193-7200

41. Margottin, F., Bour, S. P., Durand, H., Selig, L., Benichou, S., Richard, V., Thomas, D., Strebel, K., and Benarous, R. (1998) A novel human WD protein, h-beta TrCp, that interacts with HIV-1 Vpu connects CD4 to the ER degradation pathway through an F-box motif. Molecular cell 1, 565-574

42. Haller, C., Muller, B., Fritz, J. V., Lamas-Murua, M., Stolp, B., Pujol, F. M., Keppler, O. T., and Fackler, O. T. (2014) HIV-1 Nef and Vpu are functionally redundant broadspectrum modulators of cell surface receptors, including tetraspanins. J Virol 88, 1424114257

43. Hussain, A., Wesley, C., Khalid, M., Chaudhry, A., and Jameel, S. (2008) Human immunodeficiency virus type $1 \mathrm{Vpu}$ protein interacts with CD74 and modulates major histocompatibility complex class II presentation. $J$ Virol 82, 893-902

44. Lambele, M., Koppensteiner, H., Symeonides, M., Roy, N. H., Chan, J., Schindler, M., and Thali, M. (2015) Vpu is the main determinant for tetraspanin downregulation in HIV1-infected cells. $J$ Virol 89, 3247-3255

45. Liu, Y., Fu, Y., Wang, Q., Li, M., Zhou, Z., Dabbagh, D., Fu, C., Zhang, H., Li, S., Zhang, T., Gong, J., Kong, X., Zhai, W., Su, J., Sun, J., Zhang, Y., Yu, X. F., Shao, Z., Zhou, F., Wu, Y., and Tan, X. (2019) Proteomic profiling of HIV-1 infection of human CD4(+) T cells identifies PSGL-1 as an HIV restriction factor. Nat Microbiol 4, 813-825 
46. Arias, J. F., Heyer, L. N., von Bredow, B., Weisgrau, K. L., Moldt, B., Burton, D. R., Rakasz, E. G., and Evans, D. T. (2014) Tetherin antagonism by Vpu protects HIVinfected cells from antibody-dependent cell-mediated cytotoxicity. Proc Natl Acad Sci U $S$ A 111, 6425-6430

47. Callahan, M. A., Handley, M. A., Lee, Y. H., Talbot, K. J., Harper, J. W., and Panganiban, A. T. (1998) Functional interaction of human immunodeficiency virus type 1 Vpu and Gag with a novel member of the tetratricopeptide repeat protein family. J Virol 72, 5189-5197

48. Waheed, A. A., MacDonald, S., Khan, M., Mounts, M., Swiderski, M., Xu, Y., Ye, Y., and Freed, E. O. (2016) The Vpu-interacting Protein SGTA Regulates Expression of a Non-glycosylated Tetherin Species. Sci Rep 6, 24934

49. Dutta, S., and Tan, Y. J. (2008) Structural and functional characterization of human SGT and its interaction with Vpu of the human immunodeficiency virus type 1. Biochemistry 47, 10123-10131

50. Handley, M. A., Paddock, S., Dall, A., and Panganiban, A. T. (2001) Association of Vpubinding protein with microtubules and Vpu-dependent redistribution of HIV-1 Gag protein. Virology 291, 198-207

51. Forgac, M. (2007) Vacuolar ATPases: rotary proton pumps in physiology and pathophysiology. Nat Rev Mol Cell Biol 8, 917-929

52. Kane, P. M. (2006) The where, when, and how of organelle acidification by the yeast vacuolar H+-ATPase. Microbiol Mol Biol Rev 70, 177-191

53. Nishi, T., and Forgac, M. (2002) The vacuolar (H+)-ATPases--nature's most versatile proton pumps. Nat Rev Mol Cell Biol 3, 94-103

54. Arai, H., Terres, G., Pink, S., and Forgac, M. (1988) Topography and subunit stoichiometry of the coated vesicle proton pump. J Biol Chem 263, 8796-8802

55. Powell, B., Graham, L. A., and Stevens, T. H. (2000) Molecular characterization of the yeast vacuolar H+-ATPase proton pore. $J$ Biol Chem 275, 23654-23660

56. Xu, T., Vasilyeva, E., and Forgac, M. (1999) Subunit interactions in the clathrin-coated vesicle vacuolar $(\mathrm{H}(+))$-ATPase complex. J Biol Chem 274, 28909-28915

57. Finbow, M. E., Eliopoulos, E. E., Jackson, P. J., Keen, J. N., Meagher, L., Thompson, P., Jones, P., and Findlay, J. B. (1992) Structure of a $16 \mathrm{kDa}$ integral membrane protein that has identity to the putative proton channel of the vacuolar H(+)-ATPase. Protein Eng 5, 7-15

58. Leitch, B., and Finbow, M. E. (1990) The gap junction-like form of a vacuolar proton channel component appears not to be an artifact of isolation: an immunocytochemical localization study. Exp Cell Res 190, 218-226

59. Skinner, M. A., and Wildeman, A. G. (1999) beta(1) integrin binds the 16-kDa subunit of vacuolar $\mathrm{H}(+)$-ATPase at a site important for human papillomavirus E5 and plateletderived growth factor signaling. J Biol Chem 274, 23119-23127

60. Goldstein, D. J., Finbow, M. E., Andresson, T., McLean, P., Smith, K., Bubb, V., and Schlegel, R. (1991) Bovine papillomavirus E5 oncoprotein binds to the 16K component of vacuolar H(+)-ATPases. Nature 352, 347-349

61. Goldstein, D. J., Kulke, R., Dimaio, D., and Schlegel, R. (1992) A glutamine residue in the membrane-associating domain of the bovine papillomavirus type $1 \mathrm{E} 5$ oncoprotein mediates its binding to a transmembrane component of the vacuolar $\mathrm{H}(+)$-ATPase. $J$ Virol 66, 405-413

62. Kim, B. K., Nam, S. W., Min, B. S., Ban, H. S., Paik, S., Lee, K., Im, J. Y., Lee, Y., Park, J. T., Kim, S. Y., Kim, M., Lee, H., and Won, M. (2018) Bcl-2-dependent synthetic lethal interaction of the IDF-11774 with the V0 subunit C of vacuolar ATPase (ATP6V0C) in colorectal cancer. Br J Cancer 119, 1347-1357 
63. Son, S. W., Chau, G. C., Kim, S. T., and Um, S. H. (2019) Vacuolar H(+)-ATPase Subunit V0C Regulates Aerobic Glycolysis of Esophageal Cancer Cells via PKM2 Signaling. Cells $\mathbf{8}$

64. Ohkuma, S., Shimizu, S., Noto, M., Sai, Y., Kinoshita, K., and Tamura, H. (1993) Inhibition of cell growth by bafilomycin A1, a selective inhibitor of vacuolar $\mathrm{H}(+)$ ATPase. In Vitro Cell Dev Biol Anim 29A, 862-866

65. Ho, M. R., Tsai, T. T., Chen, C. L., Jhan, M. K., Tsai, C. C., Lee, Y. C., Chen, C. H., and Lin, C. F. (2017) Blockade of dengue virus infection and viral cytotoxicity in neuronal cells in vitro and in vivo by targeting endocytic pathways. Sci Rep 7, 6910

66. Sabino, C., Basic, M., Bender, D., Elgner, F., Himmelsbach, K., and Hildt, E. (2019) Bafilomycin A1 and U18666A Efficiently Impair ZIKV Infection. Viruses 11

67. Muller, K. H., Spoden, G. A., Scheffer, K. D., Brunnhofer, R., De Brabander, J. K., Maier, M. E., Florin, L., and Muller, C. P. (2014) Inhibition by cellular vacuolar ATPase impairs human papillomavirus uncoating and infection. Antimicrob Agents Chemother 58, 2905-2911

68. Suzuki, T., Yamaya, M., Sekizawa, K., Hosoda, M., Yamada, N., Ishizuka, S., Nakayama, K., Yanai, M., Numazaki, Y., and Sasaki, H. (2001) Bafilomycin A(1) inhibits rhinovirus infection in human airway epithelium: effects on endosome and ICAM-1. Am J Physiol Lung Cell Mol Physiol 280, L1115-1127

69. Jin, S., Zhang, B., Weisz, O. A., and Montelaro, R. C. (2005) Receptor-mediated entry by equine infectious anemia virus utilizes a $\mathrm{pH}$-dependent endocytic pathway. J Virol 79, 14489-14497

70. Katen, L. J., Januszeski, M. M., Anderson, W. F., Hasenkrug, K. J., and Evans, L. H. (2001) Infectious entry by amphotropic as well as ecotropic murine leukemia viruses occurs through an endocytic pathway. $J$ Virol 75, 5018-5026

71. Li, B., Clohisey, S. M., Chia, B. S., Wang, B., Cui, A., Eisenhaure, T., Schweitzer, L. D., Hoover, P., Parkinson, N. J., Nachshon, A., Smith, N., Regan, T., Farr, D., Gutmann, M. U., Bukhari, S. I., Law, A., Sangesland, M., Gat-Viks, I., Digard, P., Vasudevan, S., Lingwood, D., Dockrell, D. H., Doench, J. G., Baillie, J. K., and Hacohen, N. (2020) Genome-wide CRISPR screen identifies host dependency factors for influenza A virus infection. Nat Commun 11, 164

72. Marjuki, H., Gornitzky, A., Marathe, B. M., Ilyushina, N. A., Aldridge, J. R., Desai, G., Webby, R. J., and Webster, R. G. (2011) Influenza A virus-induced early activation of ERK and PI3K mediates V-ATPase-dependent intracellular $\mathrm{pH}$ change required for fusion. Cell Microbiol 13, 587-601

73. Muller, K. H., Kainov, D. E., El Bakkouri, K., Saelens, X., De Brabander, J. K., Kittel, C., Samm, E., and Muller, C. P. (2011) The proton translocation domain of cellular vacuolar ATPase provides a target for the treatment of influenza A virus infections. $\mathrm{Br} J$ Pharmacol 164, 344-357

74. Matsui, T., Itoh, T., and Fukuda, M. (2011) Small GTPase Rab12 regulates constitutive degradation of transferrin receptor. Traffic 12, 1432-1443

75. Iwabu, Y., Fujita, H., Kinomoto, M., Kaneko, K., Ishizaka, Y., Tanaka, Y., Sata, T., and Tokunaga, K. (2009) HIV-1 accessory protein Vpu internalizes cell-surface BST2/tetherin through transmembrane interactions leading to lysosomes. J Biol Chem 284, 35060-35072

76. Rong, L., Zhang, J., Lu, J., Pan, Q., Lorgeoux, R. P., Aloysius, C., Guo, F., Liu, S. L., Wainberg, M. A., and Liang, C. (2009) The transmembrane domain of BST-2 determines its sensitivity to down-modulation by human immunodeficiency virus type $1 \mathrm{Vpu}$. $J$ Virol 83, 7536-7546 
77. Umata, T., Moriyama, Y., Futai, M., and Mekada, E. (1990) The cytotoxic action of diphtheria toxin and its degradation in intact Vero cells are inhibited by bafilomycin A1, a specific inhibitor of vacuolar-type H(+)-ATPase. J Biol Chem 265, 21940-21945

78. Gottlinger, H. G., Dorfman, T., Cohen, E. A., and Haseltine, W. A. (1993) Vpu protein of human immunodeficiency virus type 1 enhances the release of capsids produced by gag gene constructs of widely divergent retroviruses. Proc Natl Acad Sci U S A 90, 73817385

79. Klimkait, T., Strebel, K., Hoggan, M. D., Martin, M. A., and Orenstein, J. M. (1990) The human immunodeficiency virus type 1-specific protein vpu is required for efficient virus maturation and release. J Virol 64, 621-629

80. Goffinet, C., Allespach, I., Homann, S., Tervo, H. M., Habermann, A., Rupp, D., Oberbremer, L., Kern, C., Tibroni, N., Welsch, S., Krijnse-Locker, J., Banting, G., Krausslich, H. G., Fackler, O. T., and Keppler, O. T. (2009) HIV-1 antagonism of CD317 is species specific and involves Vpu-mediated proteasomal degradation of the restriction factor. Cell host \& microbe 5, 285-297

81. Gupta, R. K., Hue, S., Schaller, T., Verschoor, E., Pillay, D., and Towers, G. J. (2009) Mutation of a single residue renders human tetherin resistant to HIV-1 Vpu-mediated depletion. PLoS Pathog 5, e1000443

82. Mangeat, B., Gers-Huber, G., Lehmann, M., Zufferey, M., Luban, J., and Piguet, V. (2009) HIV-1 Vpu neutralizes the antiviral factor Tetherin/BST-2 by binding it and directing its beta-TrCP2-dependent degradation. PLoS Pathog 5, e1000574

83. Waheed, A. A., Kuruppu, N. D., Felton, K. L., D'Souza, D., and Freed, E. O. (2014) In COS cells Vpu can both stabilize tetherin expression and counteract its antiviral activity. PloS one 9, e111628

84. Douglas, J. L., Viswanathan, K., McCarroll, M. N., Gustin, J. K., Fruh, K., and Moses, A. V. (2009) Vpu directs the degradation of the human immunodeficiency virus restriction factor BST-2/Tetherin via a $\{$ beta\} TrCP-dependent mechanism. $J$ Virol 83, 7931-7947

85. Di Domenico, F., Foppoli, C., Blarzino, C., Perluigi, M., Paolini, F., Morici, S., Coccia, R., Cini, C., and De Marco, F. (2009) Expression of human papilloma virus type 16 E5 protein in amelanotic melanoma cells regulates endo-cellular $\mathrm{pH}$ and restores tyrosinase activity. J Exp Clin Cancer Res 28, 4

86. Disbrow, G. L., Hanover, J. A., and Schlegel, R. (2005) Endoplasmic reticulum-localized human papillomavirus type $16 \mathrm{E} 5$ protein alters endosomal $\mathrm{pH}$ but not trans-Golgi $\mathrm{pH} . J$ Virol 79, 5839-5846

87. Straight, S. W., Herman, B., and McCance, D. J. (1995) The E5 oncoprotein of human papillomavirus type 16 inhibits the acidification of endosomes in human keratinocytes. $J$ Virol 69, 3185-3192

88. Thomsen, P., van Deurs, B., Norrild, B., and Kayser, L. (2000) The HPV16 E5 oncogene inhibits endocytic trafficking. Oncogene 19, 6023-6032

89. Suprynowicz, F. A., Krawczyk, E., Hebert, J. D., Sudarshan, S. R., Simic, V., Kamonjoh, C. M., and Schlegel, R. (2010) The human papillomavirus type 16 E5 oncoprotein inhibits epidermal growth factor trafficking independently of endosome acidification. $J$ Virol 84, 10619-10629

90. Pavelin, J., Reynolds, N., Chiweshe, S., Wu, G., Tiribassi, R., and Grey, F. (2013) Systematic microRNA analysis identifies ATP6V0C as an essential host factor for human cytomegalovirus replication. PLoS Pathog 9, e1003820

91. Pavelin, J., McCormick, D., Chiweshe, S., Ramachandran, S., Lin, Y. T., and Grey, F. (2017) Cellular v-ATPase is required for virion assembly compartment formation in human cytomegalovirus infection. Open Biol 7 
92. Han, L., Li, K., Jin, C., Wang, J., Li, Q., Zhang, Q., Cheng, Q., Yang, J., Bo, X., and Wang, S. (2017) Human enterovirus 71 protein interaction network prompts antiviral drug repositioning. Sci Rep 7, 43143

93. Duan, X., Lu, X., Li, J., and Liu, Y. (2008) Novel binding between pre-membrane protein and vacuolar ATPase is required for efficient dengue virus secretion. Biochem Biophys Res Commun 373, 319-324

94. Geyer, M., Yu, H., Mandic, R., Linnemann, T., Zheng, Y. H., Fackler, O. T., and Peterlin, B. M. (2002) Subunit H of the V-ATPase binds to the medium chain of adaptor protein complex 2 and connects Nef to the endocytic machinery. J Biol Chem 277, 28521-28529

95. Lu, X., Yu, H., Liu, S. H., Brodsky, F. M., and Peterlin, B. M. (1998) Interactions between HIV1 Nef and vacuolar ATPase facilitate the internalization of CD4. Immunity 8, 647-656

96. Mandic, R., Fackler, O. T., Geyer, M., Linnemann, T., Zheng, Y. H., and Peterlin, B. M. (2001) Negative factor from SIV binds to the catalytic subunit of the V-ATPase to internalize CD4 and to increase viral infectivity. Mol Biol Cell 12, 463-473

97. Franchini, G., Mulloy, J. C., Koralnik, I. J., Lo Monico, A., Sparkowski, J. J., Andresson, T., Goldstein, D. J., and Schlegel, R. (1993) The human T-cell leukemia/lymphotropic virus type I p12I protein cooperates with the E5 oncoprotein of bovine papillomavirus in cell transformation and binds the 16-kilodalton subunit of the vacuolar H+ ATPase. $J$ Virol 67, 7701-7704

98. Koralnik, I. J., Mulloy, J. C., Andresson, T., Fullen, J., and Franchini, G. (1995) Mapping of the intermolecular association of human $\mathrm{T}$ cell leukaemia/lymphotropic virus type I p12I and the vacuolar H+-ATPase $16 \mathrm{kDa}$ subunit protein. J Gen Virol 76 ( Pt 8), 19091916

99. Nguyen, K. L., llano, M., Akari, H., Miyagi, E., Poeschla, E. M., Strebel, K., and Bour, S. (2004) Codon optimization of the HIV-1 vpu and vif genes stabilizes their mRNA and allows for highly efficient Rev-independent expression. Virology 319, 163-175

100. Adachi, A., Gendelman, H. E., Koenig, S., Folks, T., Willey, R., Rabson, A., and Martin, M. A. (1986) Production of acquired immunodeficiency syndrome-associated retrovirus in human and nonhuman cells transfected with an infectious molecular clone. J Virol 59, 284-291

101. Miyagi, E., Andrew, A. J., Kao, S., and Strebel, K. (2009) Vpu enhances HIV-1 virus release in the absence of Bst-2 cell surface down-modulation and intracellular depletion. Proc Natl Acad Sci U S A 106, 2868-2873

102. Miller, J., and Stagljar, I. (2004) Using the yeast two-hybrid system to identify interacting proteins. Methods in molecular biology 261, 247-262

103. Waheed, A. A., Ablan, S. D., Mankowski, M. K., Cummins, J. E., Ptak, R. G., Schaffner, C. P., and Freed, E. O. (2006) Inhibition of HIV-1 replication by amphotericin B methyl ester: selection for resistant variants. J Biol Chem 281, 28699-28711

104. Freed, E. O., and Martin, M. A. (1994) Evidence for a functional interaction between the V1/V2 and C4 domains of human immunodeficiency virus type 1 envelope glycoprotein gp120. J Virol 68, 2503-2512 


\section{Figures Legends}

Fig. 1. ATP6V0C co-immunoprecipitates with Vpu. 293T cells were transfected with vectors expressing FLAG-tagged ATP6V0C or Vpu alone or in combination. Twenty-four h posttransfection, cell lysates were prepared and immunoprecipitated with agarose beads conjugated with anti-FLAG (A) or anti-Vpu (B) antibodies. Complexes were washed and both cell lysates and immunoprecipitated samples were subjected to immunoblotting with anti-FLAG and antiVpu antibodies to detect FLAG-tagged ATP6V0C and Vpu, respectively.

Fig. 2. ATP6V0C overexpression has no effect on HIV-1 release. (A) 293 T cells were transfected with WT or Vpu-defective (delVpu) pNL4-3 HIV-1 molecular clones with or without vectors expressing FLAG-tagged ATP6V0C and HA-tagged human tetherin. One day posttransfection, cell and viral lysates were prepared and subjected to western blot analysis with HIV-Ig to detect the Gag precursor Pr55Gag (Pr55) and the CA protein p24, or anti-FLAG to detect FLAG-tagged ATP6V0C, anti-HA to detect HA-tagged tetherin, or antisera against Vpu or tubulin. Mobility of molecular mass standards is shown on the right of the anti-HA blot. (B) Virus release efficiency (VRE) was calculated as the amount of virion-associated p24 (CA) relative to total Gag in cell and virus. VRE was set to $100 \%$ for WT pNL4-3 in the absence of ATP6V0C and tetherin. Data shown are \pm SD from three independent experiments.

Fig. 3. Overexpression of ATP6V0C increases the levels of tetherin but reduces CD4 and TR expression. 293T cells were transfected with vectors expressing (A) HA-tagged tetherin without or with FLAG-tagged ATP6V0C and Vpu expression vectors; (B) CD4 without or with FLAG-tagged ATP6V0C and Vpu expression vectors; (C) HA-tagged tetherin without or with FLAG-tagged ATP6V0C or ATP6V0C" expression vectors; (D) transferrin receptor (TR) without or with FLAG-tagged ATP6V0C or ATP6V0C" expression vectors. Twenty-four h posttransfection, cell lysates were prepared and subjected to western blot analysis with anti-FLAG to detect FLAG-tagged ATP6V0C, anti-HA to detect HA-tagged tetherin, or antisera against Vpu, CD4, or TR. Molecular mass markers are shown on the right of the anti-HA blots.

Fig. 4. Tetherin co-immunoprecipitates with ATP6V0C independent of Vpu. 293T cells were transfected with vectors expressing FLAG-tagged ATP6V0C without or with HA-tagged tetherin and Vpu expression vectors (A) or HA-tagged tetherin without or with FLAG-tagged ATP6V0C and Vpu expression vectors (B). Twenty-four h post-transfection, cell lysates were prepared and immunoprecipitated with agarose beads conjugated with anti-FLAG (A) or anti-HA (B) antibodies. Complexes were washed and both cell lysates and immunoprecipitated samples were subjected to immunoblotting with anti-FLAG, anti-HA and anti-Vpu antibodies to detect FLAGtagged ATP6V0C, HA-tagged tetherin, and Vpu, respectively. Mobility of molecular mass standards is shown on the right of the anti-HA blot. The location of different species of tetherin and $\operatorname{Ig}$ light chain $(\mathrm{IgL})$ is indicated by the arrows.

Fig. 5. ATP6V0C overexpression stabilizes tetherin. (A) $293 \mathrm{~T}$ cells were transfected with vectors expressing HA-tagged tetherin without or with FLAG-tagged ATP6V0C expression vector. $24 \mathrm{~h}$ post-transfection, cells were labeled with $\left[{ }^{35} \mathrm{~S}\right] \mathrm{Met} / \mathrm{Cys}$ for $30 \mathrm{~min}$ and then chased for $0,0.5,1,2$, or $4 \mathrm{~h}$ in unlabeled medium. Cell lysates were prepared and immunoprecipitated with anti-HA antibodies and analyzed by SDS-PAGE followed by fluorography. Positions of 26$\mathrm{kDa}$ (glycosylated) and 23-kDa (non-glycosylated) tetherin are indicated. (B) The levels of radiolabeled 23- and 26-kDa tetherin species were quantified at each time point from three independent experiments, \pm SD. Tetherin levels at time zero was taken as $100 \%$. The $p$ values (two-tailed paired t-test) are calculated for each time point. ${ }^{*} \mathrm{p}<0.05,{ }^{*} \mathrm{p}<0.02$. The half-life $\left(t_{1 / 2}\right)$ of tetherin under each condition is indicated. 
Fig. 6. The cytoplasmic tail, GPI-anchor, and dimerization of human tetherin are required for ATP6V0C-mediated tetherin stabilization. 293T cells were transfected with vectors expressing HA-tagged tetherin with or without FLAG-tagged ATP6V0C expression vector. Tetherin variants tested include: (A) a cytoplasmic tail deletion mutant (delCT), a GPI-anchor deletion mutant (delGPI), non-glycosylated mutant (NN), and non-dimerizing mutant (CCC); (B) Agm tetherin (Agm), Rhesus tetherin (Rh), and a human tetherin containing the Agm (Hu-Agm) or $\mathrm{Rh}(\mathrm{Hu}-\mathrm{Rh})$ transmembrane domains. One day posttransfection, cell lysates were prepared and immunoblotted with anti-HA to detect HA-tagged tetherin, anti-FLAG to detect FLAG-tagged ATP6V0C, or anti-tubulin antisera. Mobility of molecular mass standards is shown on the right of the anti-HA blot.

Fig. 7. Stabilization of tetherin is not due to inhibition of lysosomal degradation. 293T cells were transfected with vectors expressing HA-tagged tetherin $(\mathbf{A})$ or $\mathrm{Vpu}(\mathbf{B})$ without or with FLAG-tagged ATP6V0C expression vector. Eight h posttransfection, cells were either untreated or treated with bafilomycin for $18 \mathrm{~h}$ prior to cell lysis and immunoblotting with anti-HA to detect HA-tagged tetherin, anti-FLAG to detect FLAG-tagged ATP6V0C, or anti-TR or anti-tubulin antisera. Molecular mass markers are shown on the right of the anti-HA blot.

Fig. 8. ATP6V0C overexpression sequesters tetherin in LAMP-1 and CD63-positive compartments. 293T cells were transfected with HA-tagged tetherin expression vector alone or in combination with the FLAG-tagged ATP6V0C expression vector. Twenty-four h posttransfection cells were fixed and stained with (A) anti-HA (green), anti-FLAG (red) and DAPI (blue); (B) anti-HA (green), anti-CD63 (red) and anti-FLAG (blue); (C) anti-HA (green), antiLAMP-1 (red) and anti-FLAG (blue) antibodies. Images were acquired with a Delta-Vision RT deconvolution microscope. Colocalization between tetherin and ATP6V0C (A), tetherin and CD63 (B), and tetherin and LAMP-1 (C) was quantified by calculating the Pearson correlation coefficient (R values) \pm SD from 20-30 cells. Scale bars, $15 \mu \mathrm{m}$.

Fig. 9. The knockdown of ATP6V0C inhibits HIV-1 release by enhancing tetherin expression. HeLa (A and B) or 293T (D and E) cells were transfected with non-targeting siRNA (NT) or ATP6V0C siRNA alone or in combination with tetherin siRNA. One-day later, cells were infected with VSV-G-pseudotyped wild-type (WT) or Vpu-defective HIV-1; after $8 \mathrm{~h}$ the medium was changed, and the siRNA transfection was repeated. Two days later, cell and viral lysates were prepared and subjected to western blot analysis with HIV-Ig to detect the Gag precursor Pr55Gag (Pr55) and the CA protein p24, or antibodies specific for tetherin, Vpu, or tubulin. (B and E), virus release efficiency (VRE) was calculated as in Fig. 2. VRE was set to $100 \%$ for WT HIV-1 in the presence of NT siRNA. (C) To monitor ATP6V0C knockdown efficiency, 293T cells were transfected with the FLAG-tagged ATP6V0C expression vector and treated with NT or ATP6V0C siRNA as in Fig. 9A and two-days later cells were lysed. Relative expression of FLAG-tagged ATP6V0C in the presence of NT siRNA was set to $100 \%$. Mobility of molecular mass standards is indicated on the right of the tetherin blot. Data shown are \pm SD from three independent experiments. P values (two-tailed paired t-test): ${ }^{*} \mathrm{p}<0.02,{ }^{* *} \mathrm{p}<0.01,{ }^{* * *} \mathrm{p}<0.002$

Fig. S1. Localization of tetherin mutants in the presence of ATP6V0C. 293T cells were transfected with HA-tagged mutant tetherin expression vector alone or in combination with the FLAG-tagged ATP6V0C expression vector. Tetherin mutants tested include: (S1A) a cytoplasmic tail deletion mutant (delCT); (S1B) a non-dimerizing mutant (CCC); (S1C) a GPI-anchor deletion mutant (delGPI); and (S1D) a non-glycosylated mutant (NN). Twenty-four h post-transfection cells were fixed, stained as in Fig. 8A and images were acquired with a Delta-Vision RT deconvolution microscope. 
A

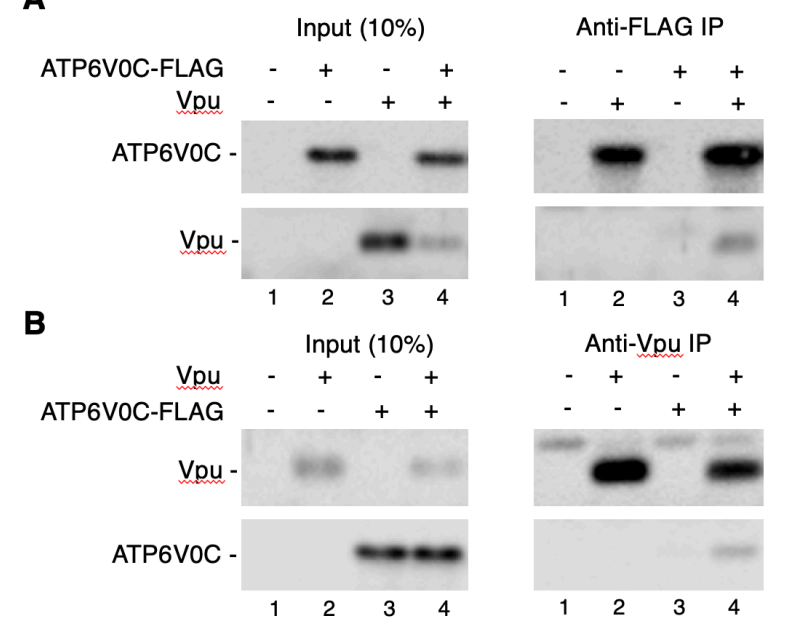

Fig. 1. ATP6V0C co-immunoprecipitates with Vpu. 293T cells were transfected with vectors expressing FLAG-tagged ATP6V0C or Vpu alone or in combination. Twenty-four h post-transfection, cell lysates were prepared and immunoprecipitated with agarose beads conjugated with anti-FLAG (A) or anti-Vpu (B) antibodies. Complexes were washed and both cell lysates and immunoprecipitated samples were subjected to immunoblotting with anti-FLAG and anti-Vpu antibodies to detect FLAG-tagged ATP6V0C and Vpu, respectively. 
A
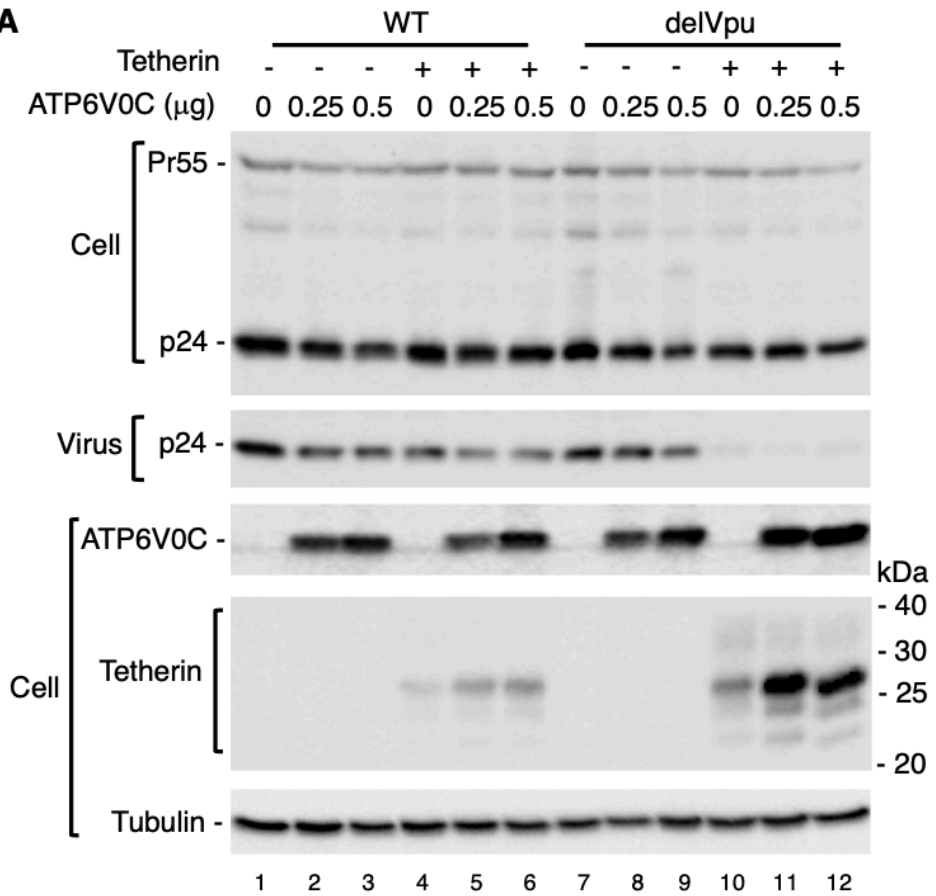

B

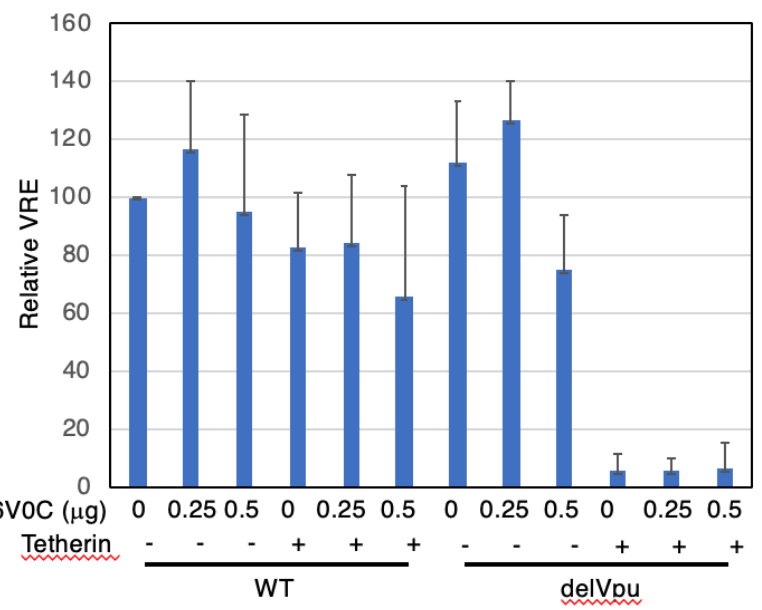

Fig. 2. ATP6V0C overexpression has no effect on HIV-1 release. (A) 293T cells were transfected with WT or Vpu-defective (delVpu) pNL4-3 HIV-1 molecular clones with or without vectors expressing FLAG-tagged ATP6V0C and HA-tagged human tetherin. One day posttransfection, cell and viral lysates were prepared and subjected to western blot analysis with HIV-Ig to detect the Gag precursor Pr55Gag (Pr55) and the CA protein p24, or anti-FLAG to detect FLAG-tagged ATP6V0C, anti-HA to detect HAtagged tetherin, or antisera against Vpu or tubulin. Mobility of molecular mass standards is shown on the right of the anti-HA blot. (B) Virus release efficiency (VRE) was calculated as the amount of virionassociated p24 (CA) relative to total Gag in cell and virus. VRE was set to 100\% for WT pNL4-3 in the absence of ATP6V0C and tetherin. Data shown are \pm SD from three independent experiments. 
A

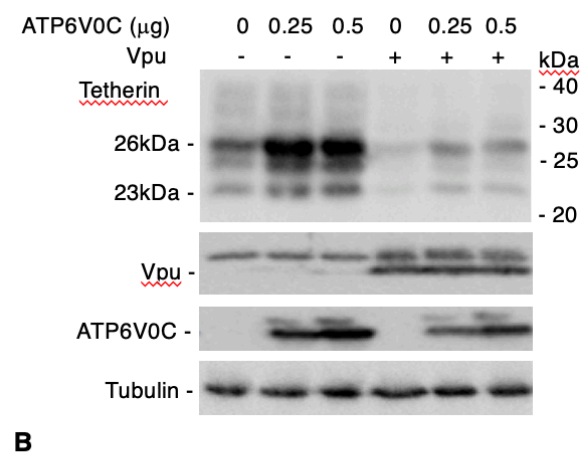

B

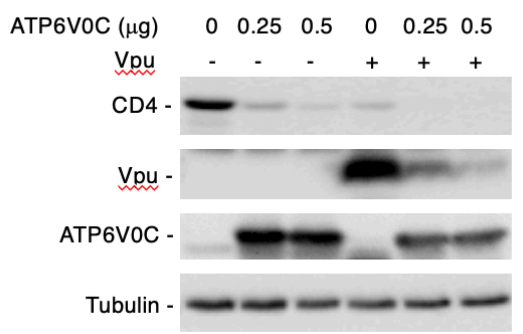

C
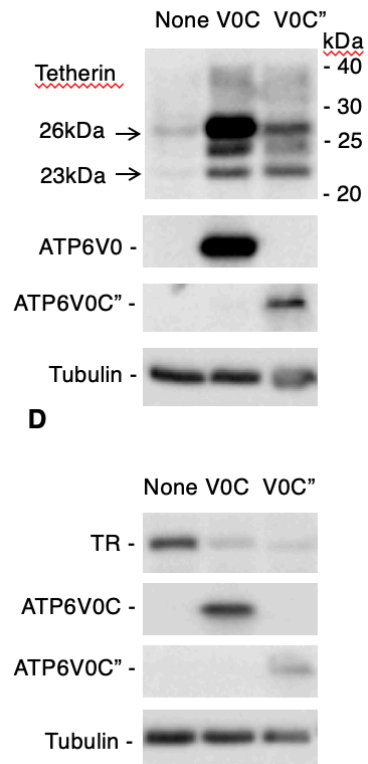

Fig. 3. Overexpression of ATP6VOC increases the levels of tetherin but reduces CD4 and TR expression. 293T cells were transfected with vectors expressing (A) HA-tagged tetherin without or with FLAG-tagged ATP6V0C and Vpu expression vectors; (B) CD4 without or with FLAG-tagged ATP6V0C and Vpu expression vectors; (C) HA-tagged tetherin without or with FLAG-tagged ATP6V0C or ATP6V0C" expression vectors; (D) transferrin receptor (TR) without or with FLAG-tagged ATP6V0C or ATP6V0C" expression vectors. Twenty-four h post-transfection, cell lysates were prepared and subjected to western blot analysis with anti-FLAG to detect FLAG-tagged ATP6V0C, anti-HA to detect HA-tagged tetherin, or antisera against $\mathrm{Vpu}, \mathrm{CD} 4$, or TR. Molecular mass markers are shown on the right of the antiHA blots. 


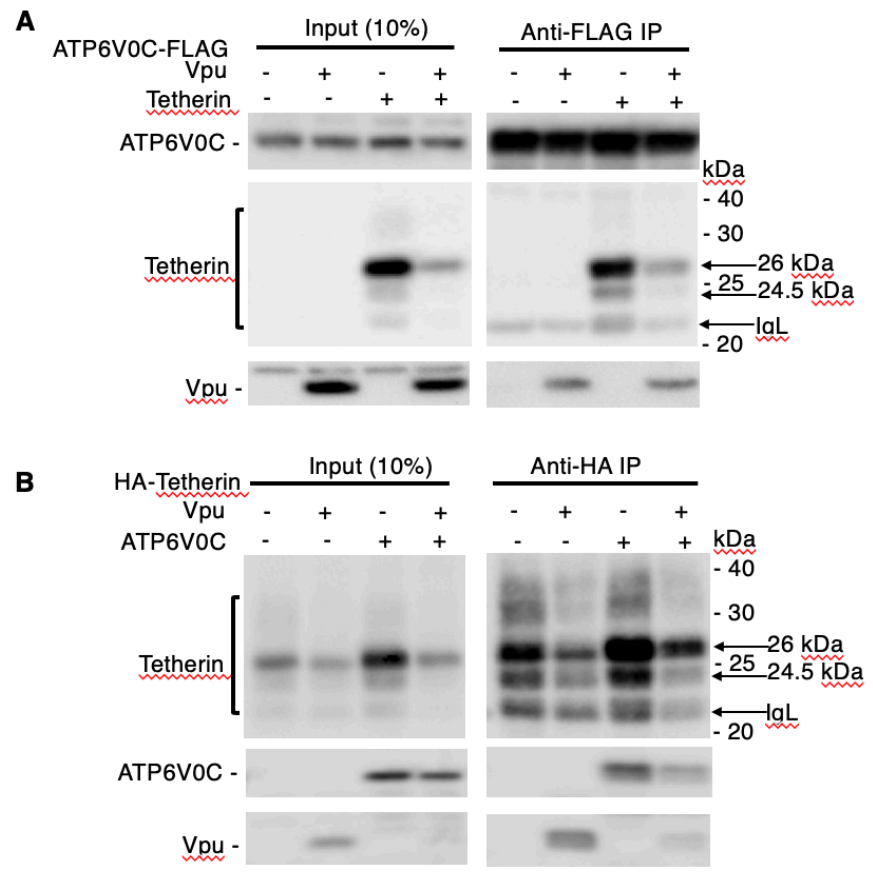

Fig. 4. Tetherin co-immunoprecipitates with ATP6V0C independent of Vpu. 293T cells were transfected with vectors expressing FLAG-tagged ATP6V0C without or with HA-tagged tetherin and Vpu expression vectors (A) or HA-tagged tetherin without or with FLAG-tagged ATP6V0C and Vpu expression vectors (B). Twenty-four h post-transfection, cell lysates were prepared and immunoprecipitated with agarose beads conjugated with anti-FLAG (A) or anti-HA (B) antibodies. Complexes were washed and both cell lysates and immunoprecipitated samples were subjected to immunoblotting with anti-FLAG, anti-HA and anti-Vpu antibodies to detect FLAG-tagged ATP6V0C, HA-tagged tetherin, and Vpu, respectively. Mobility of molecular mass standards is shown on the right of the anti-HA blot. The location of different species of tetherin and $\operatorname{Ig}$ light chain (IgL) is indicated by the arrows. 

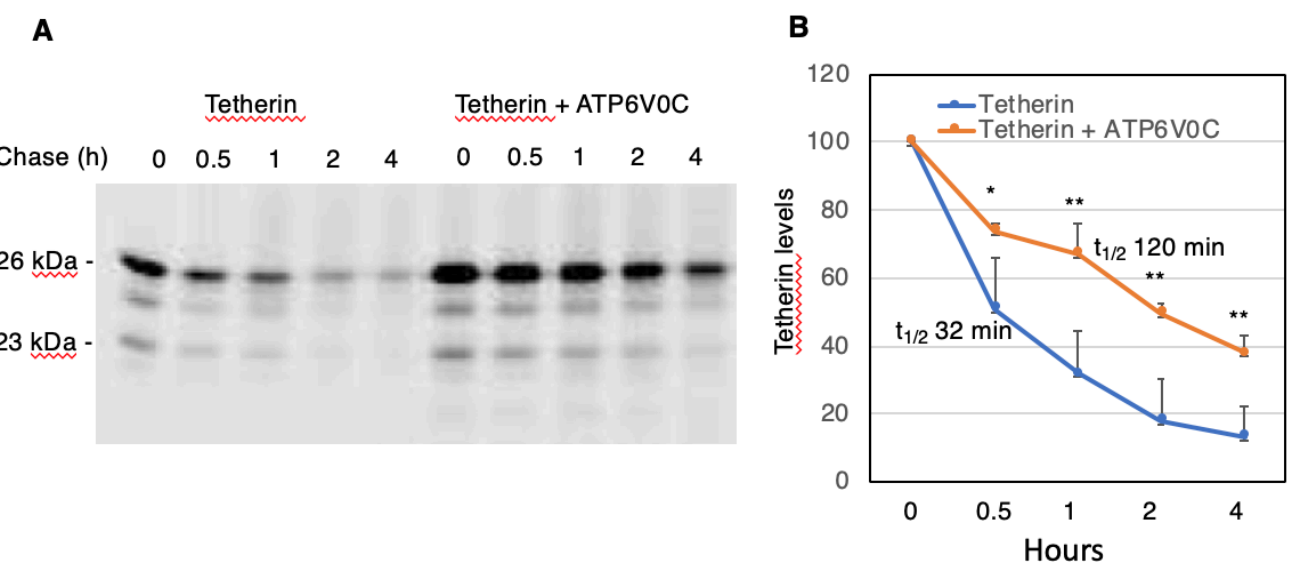

Fig. 5. ATP6V0C overexpression stabilizes tetherin. (A) 293T cells were transfected with vectors expressing HA-tagged tetherin without or with FLAG-tagged ATP6V0C expression vector. $24 \mathrm{~h}$ posttransfection, cells were labeled with $\left[{ }^{35} \mathrm{~S}\right] \mathrm{Met} / \mathrm{Cys}$ for $30 \mathrm{~min}$ and then chased for $0,0.5,1,2$, or $4 \mathrm{~h}$ in unlabeled medium. Cell lysates were prepared and immunoprecipitated with anti-HA antibodies and analyzed by SDS-PAGE followed by fluorography. Positions of 26-kDa (glycosylated) and 23-kDa (nonglycosylated) tetherin are indicated. (B) The levels of radiolabeled 23- and 26-kDa tetherin species were quantified at each time point from three independent experiments, \pm SD. Tetherin levels at time zero was taken as $100 \%$. The $\mathrm{p}$ values (two-tailed paired t-test) are calculated for each time point. ${ }^{*} \mathrm{p}<0.05, * * \mathrm{p}<$ 0.02. The half-life $\left(\mathrm{t}_{1 / 2}\right)$ of tetherin under each condition is indicated. 
A

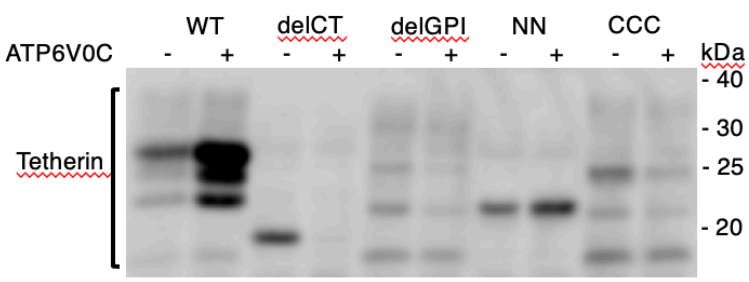

ATP6VOC -

Tubulin -
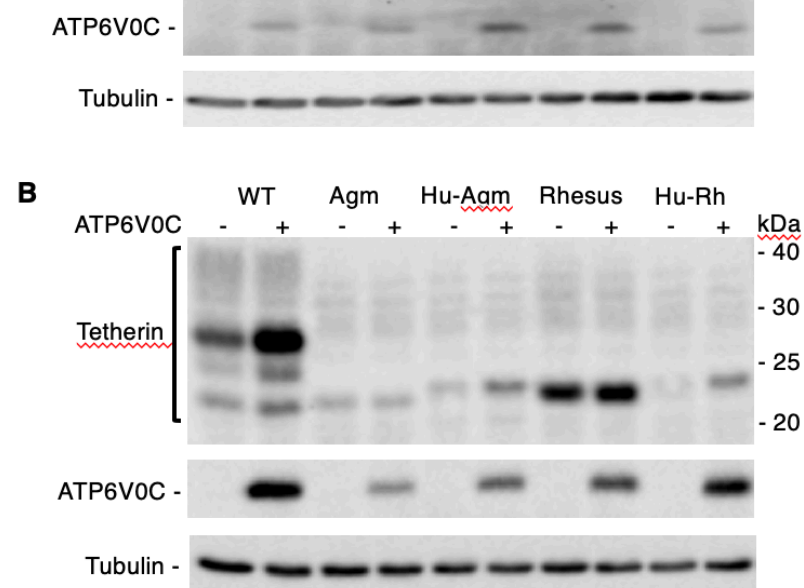

Fig. 6. The cytoplasmic tail, GPI-anchor, and dimerization of human tetherin are required for ATP6V0C-mediated tetherin stabilization. 293T cells were transfected with vectors expressing HAtagged tetherin with or without FLAG-tagged ATP6V0C expression vector. Tetherin variants tested include: (A) a cytoplasmic tail deletion mutant (delCT), a GPI-anchor deletion mutant (delGPI), nonglycosylated mutant (NN), and non-dimerizing mutant (CCC); (B) Agm tetherin (Agm), Rhesus tetherin $(\mathrm{Rh})$, and a human tetherin containing the Agm (Hu-Agm) or $\mathrm{Rh}(\mathrm{Hu}-\mathrm{Rh})$ transmembrane domains. One day posttransfection, cell lysates were prepared and immunoblotted with anti-HA to detect HA-tagged tetherin, anti-FLAG to detect FLAG-tagged ATP6V0C, or anti-tubulin antisera. Mobility of molecular mass standards is shown on the right of the anti-HA blot. 
A

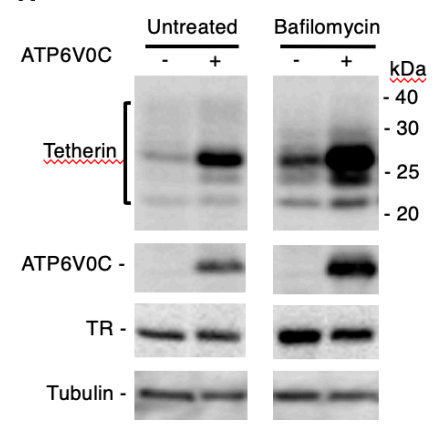

B

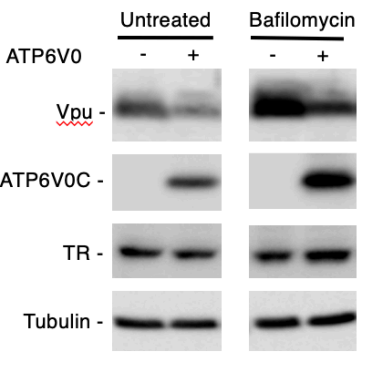

Fig. 7. Stabilization of tetherin is not due to inhibition of lysosomal degradation. 293T cells were transfected with vectors expressing HA-tagged tetherin (A) or Vpu (B) without or with FLAG-tagged ATP6V0C expression vector. Eight $h$ posttransfection, cells were either untreated or treated with bafilomycin for $18 \mathrm{~h}$ prior to cell lysis and immunoblotting with anti-HA to detect HA-tagged tetherin, anti-FLAG to detect FLAG-tagged ATP6V0C, or anti-TR or anti-tubulin antisera. Molecular mass markers are shown on the right of the anti-HA blot. 
bioRxiv preprint doi: https://doi.org/10.1101/2020.03.01.972125; this version posted March 3, 2020. The copyright holder for this preprint (which was not certified by peer review) is the author/funder. This article is a US Government work. It is not subject to copyright under 17 USC 105 and is also made available for use under a CCO license.
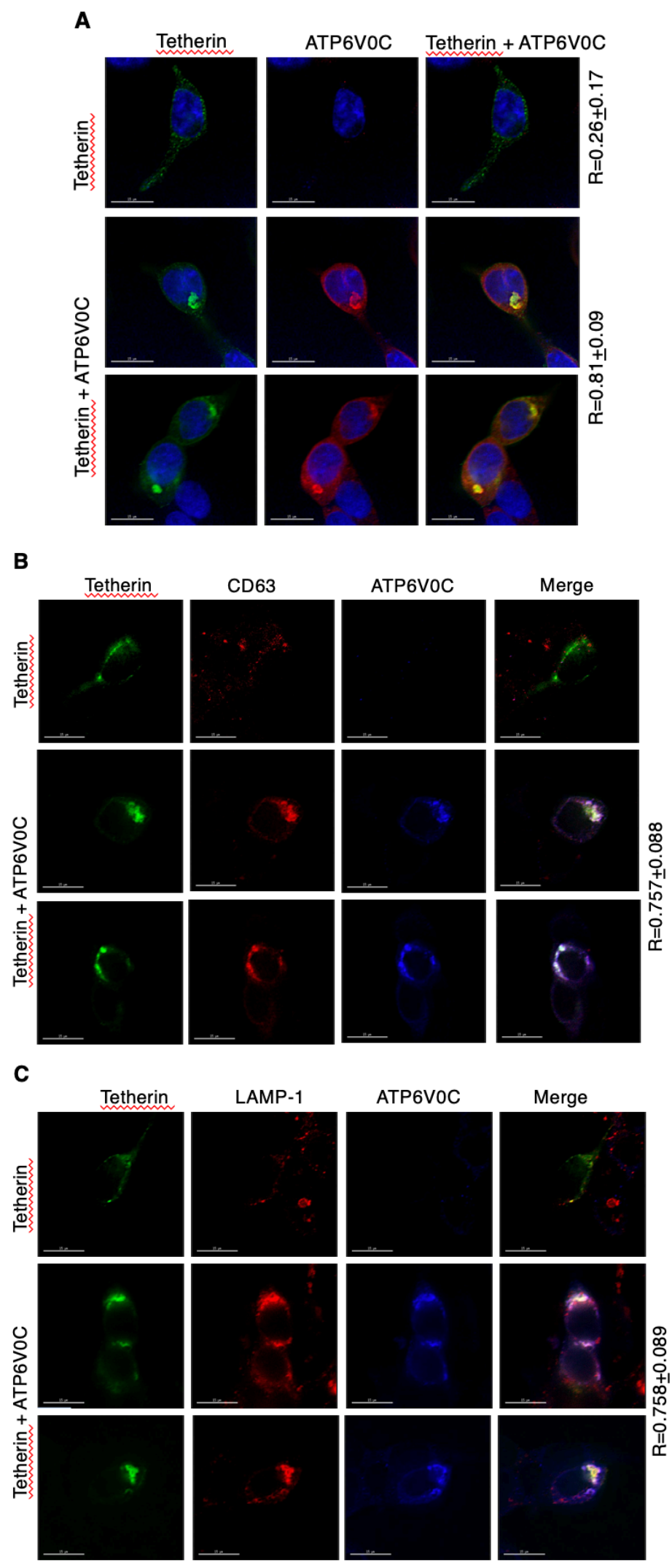
Fig. 8. ATP6V0C overexpression sequesters tetherin in LAMP-1 and CD63-positive compartments. 293 T cells were transfected with HA-tagged tetherin expression vector alone or in combination with the FLAG-tagged ATP6V0C expression vector. Twenty-four h post-transfection cells were fixed and stained with (A) anti-HA (green), anti-FLAG (red) and DAPI (blue); (B) anti-HA (green), anti-CD63 (red) and anti-FLAG (blue); (C) anti-HA (green), anti-LAMP-1 (red) and anti-FLAG (blue) antibodies. Images were acquired with a Delta-Vision RT deconvolution microscope. Colocalization between tetherin and ATP6V0C (A), tetherin and CD63 (B), and tetherin and LAMP-1 (C) was quantified by calculating the Pearson correlation coefficient (R values) \pm SD from 20-30 cells. Scale bars, $15 \mu \mathrm{m}$. 


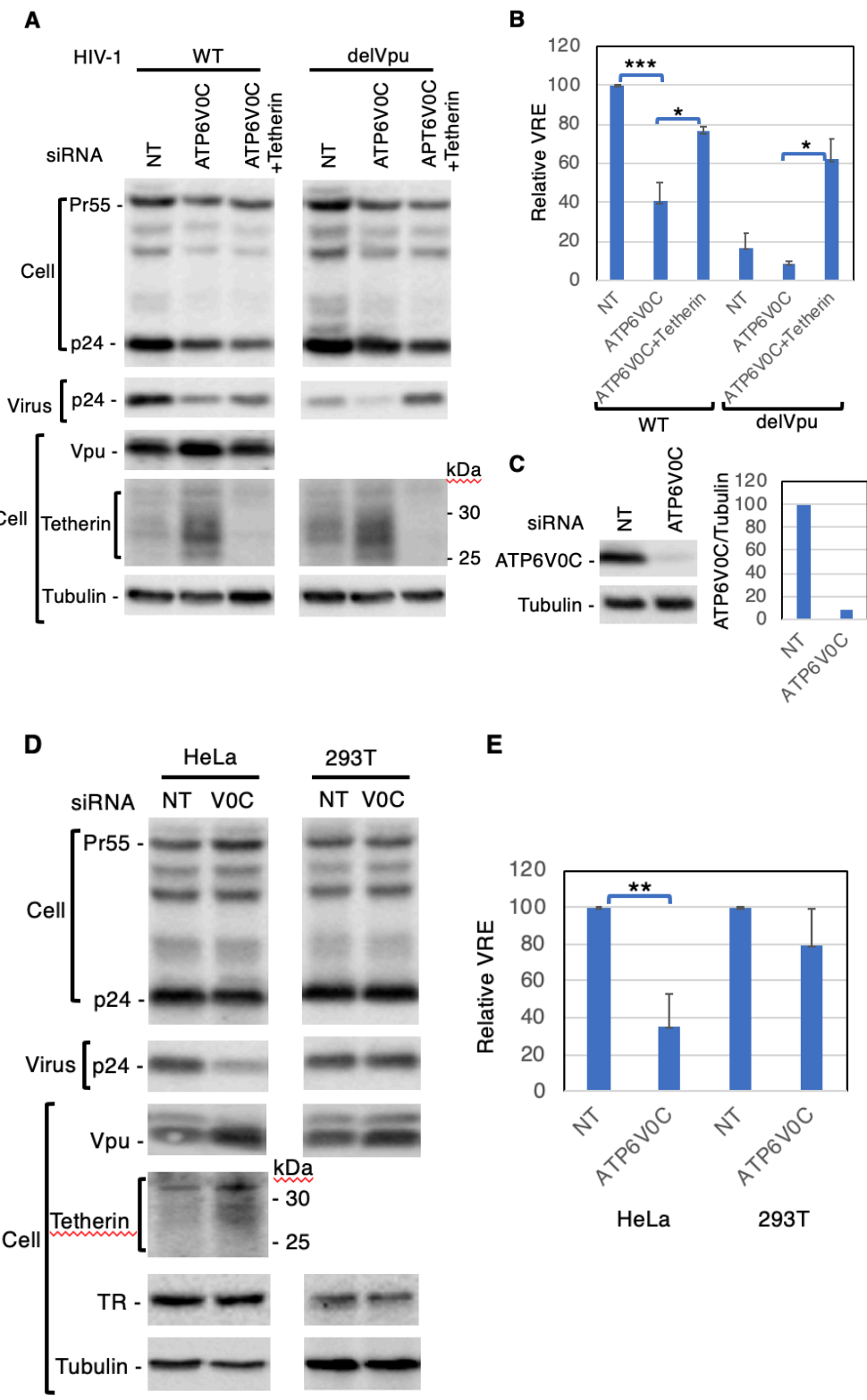

Fig. 9. The knockdown of ATP6VOC inhibits HIV-1 release by enhancing tetherin expression. HeLa (A and B) or 293T (D and E) cells were transfected with non-targeting siRNA (NT) or ATP6V0C siRNA alone or in combination with tetherin siRNA. One-day later, cells were infected with VSV-Gpseudotyped wild-type (WT) or Vpu-defective HIV-1; after $8 \mathrm{~h}$ the medium was changed, and the siRNA transfection was repeated. Two days later, cell and viral lysates were prepared and subjected to western blot analysis with HIV-Ig to detect the Gag precursor Pr55Gag (Pr55) and the CA protein p24, or antibodies specific for tetherin, Vpu, or tubulin. (B and E), virus release efficiency (VRE) was calculated as in Fig. 2. VRE was set to $100 \%$ for WT HIV-1 in the presence of NT siRNA. (C) To monitor ATP6V0C knockdown efficiency, 293T cells were transfected with the FLAG-tagged ATP6V0C expression vector and treated with NT or ATP6V0C siRNA as in Fig. 9A and two-days later cells were lysed. Relative expression of FLAG-tagged ATP6V0C in the presence of NT siRNA was set to $100 \%$. Mobility of molecular mass standards is indicated on the right of the tetherin blot. Data shown are \pm SD from three to four independent experiments. P values (two-tailed paired t-test): ${ }^{*} \mathrm{p}<0.02,{ }^{*} \mathrm{p}<0.01$, $* * * \mathrm{p}<0.002$. 
A

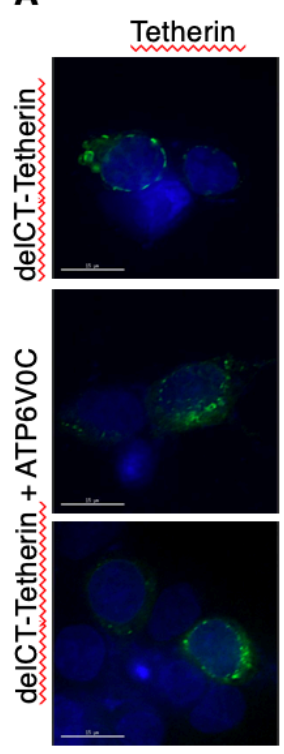

C
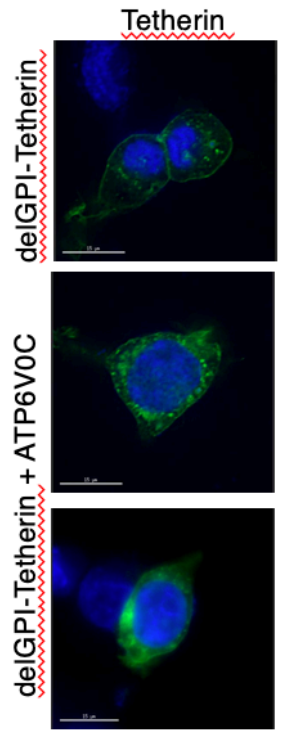

ATP6V0C

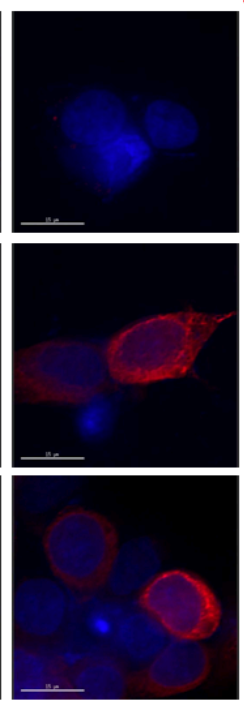

ATP6VOC
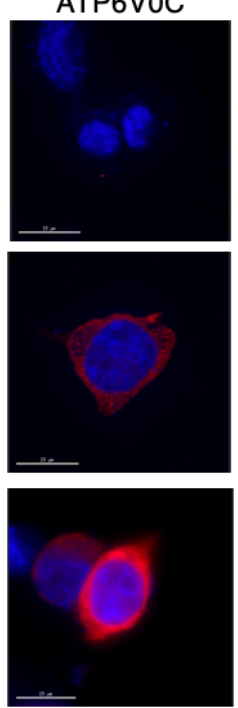

B
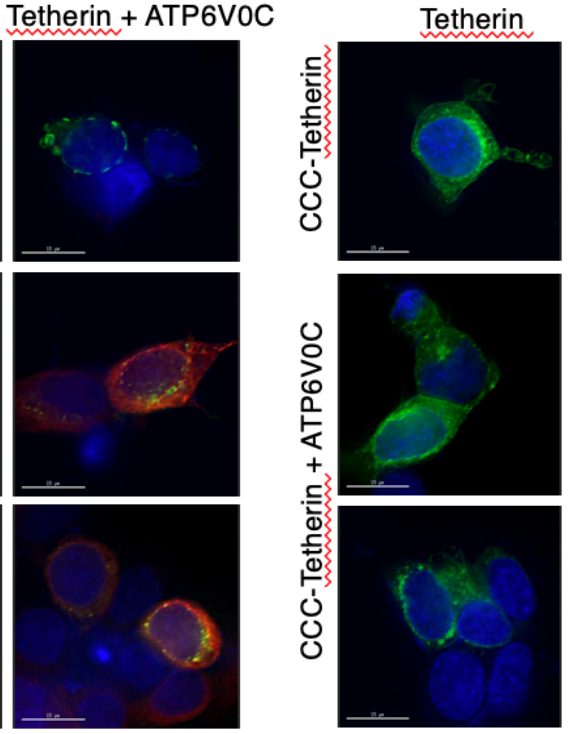

D
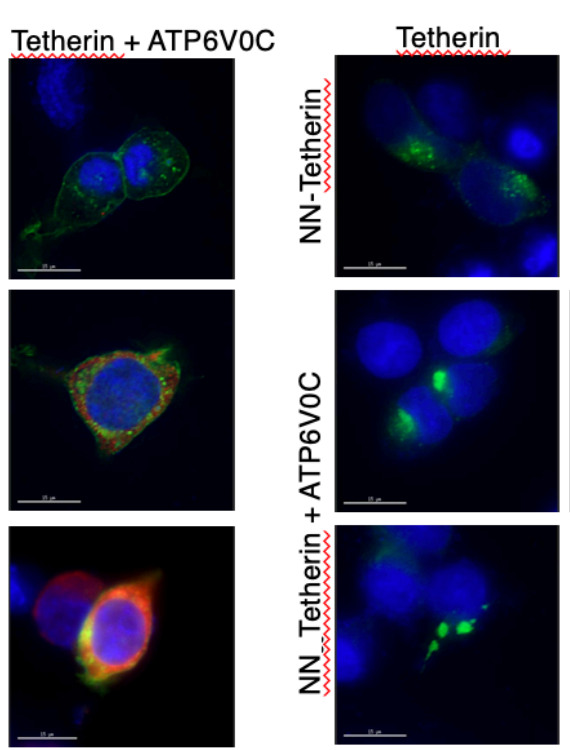

ATP6V0C

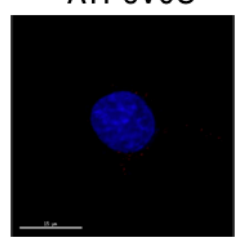

Tetherin + ATP6V0C
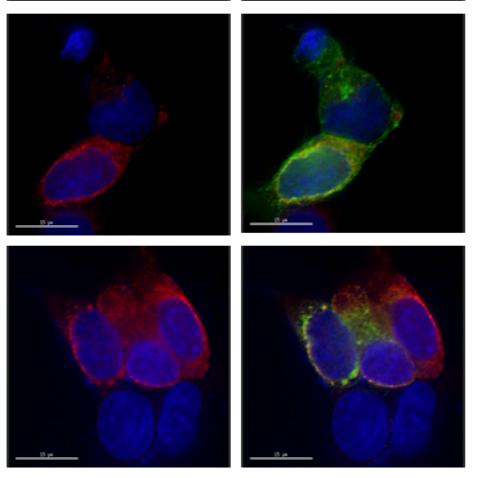

ATP6VOC

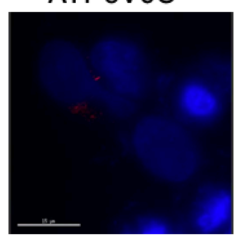

Tetherin + ATP6V0C
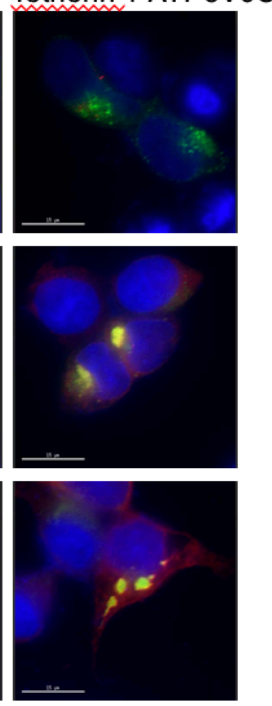

Fig. S1. Localization of tetherin mutants in the presence of ATP6V0C. 293T cells were transfected with HA-tagged mutant tetherin expression vector alone or in combination with the FLAG-tagged ATP6V0C expression vector. Tetherin mutants tested include: (S1A) a cytoplasmic tail deletion mutant (delCT); (S1B) a non-dimerizing mutant (CCC); (S1C) a GPI-anchor deletion mutant (delGPI); and (S1D) a non-glycosylated mutant (NN). Twenty-four h post-transfection cells were fixed, stained as in Fig. 8A and images were acquired with a Delta-Vision RT deconvolution microscope. Scale bars, $15 \mu \mathrm{m}$. 